\title{
Second order rectifiability of integral varifolds of locally bounded first variation
}

\author{
Ulrich Menne*
}

November 4, 2018

\begin{abstract}
It is shown that every integral varifold in an open subset of Euclidean space whose first variation with respect to area is representable by integration can be covered by a countable collection of submanifolds of the same dimension of class 2 and that their mean curvature agrees almost everywhere with the variationally defined generalised mean curvature of the varifold.
\end{abstract}

2000 Mathematics Subject Classification. Primary 49Q15; Secondary 35J60.

\section{Introduction}

Overview In the present paper the existence of an approximate second order structure for integral varifolds in Euclidean space whose first variation with respect to area is representable by integration is established. Such varifolds are called "of locally bounded first variation" in [Sim83]. Moreover, it is proven that the variationally defined generalised mean curvature of the varifold agrees almost everywhere with the mean curvature induced from the approximate second order structure. This problem can be considered a geometric, nonlinear, higher multiplicity version of the following linear one: Prove existence of approximate second order differentials for weakly differentiable functions whose distributional Laplacian is representable by integration (i.e., by a "vector-valued Radon measure") and show that these differentials satisfy the equation Lebesgue almost everywhere. Clearly, the linear case itself is not too hard to solve, and in fact follows immediately from classical results if the distributional Laplacian is integrable with respect to Lebesgue measure to a power larger than 1 . Nevertheless, the main objective of the present paper is to develop a method which is based on the study of the nearly linear case and is sufficiently robust to be applied to the present elliptic system of geometric partial differential equations involving higher multiplicity.

Results of the type obtained in the present paper have proven useful for example in the context of Brakke's mean curvature flow or sharp and diffuse interfaces or image reconstruction or the Willmore functional, see Bra78, Sch01, Rög04, RS06, MR09, AM03, Sch09] and the references therein.

*The author acknowledges financial support via the DFG Forschergruppe 469. The major part of this work was accomplished while the author was at the University of Tübingen. Some parts were done at the ETH Zürich and the work was put in its final form at the AEI Golm. AEI publication number. AEI-2008-065 
Result of the present paper in the context of known results Fix positive integers $m$ and $n$ with $m<n$. The principal result is as follows, see Section 1 for the notation used.

Theorem 1 (see 3.6). Suppose $U$ is an open subset of $\mathbf{R}^{n}, V \in \mathbf{I V}_{m}(U)$ and $\|\delta V\|$ is a Radon measure.

Then there exists a countable collection $C$ of $m$ dimensional submanifolds of $\mathbf{R}^{n}$ of class 2 such that $\|V\|(U \sim \bigcup C)=0$ and each member $M$ of $C$ satisfies

$$
\mathbf{h}(V ; z)=\mathbf{h}(M ; z) \quad \text { for }\|V\| \text { almost all } z \in U \cap M .
$$

In the terminology of Anzellotti and Serapioni AS94, 3.1] the first part of the conclusion can be expressed equivalently by the condition that $U \cap$ $\left\{z: 0<\boldsymbol{\Theta}^{m}(\|V\|, z)<\infty\right\}$ meets every compact subset of $U$ in a set which is $\left(\mathscr{H}^{m}, m\right)$ rectifiable of class $\mathscr{C}^{2}$. The second part of the assertion is sometimes called "locality of the mean curvature", see Schätzle Sch09, §4].

Theorem 1 contains (and reproves) the fact that $\mathbf{h}(V ; z) \in \operatorname{Nor}^{m}(\|V\|, z)$ for $\|V\|$ almost all $z$ previously obtained by Brakke [Bra78, 5.8], see 3.7. Moreover, it is worth noting, see 3.8, that if $V$ is a curvature varifold with boundary in $U$ in the sense of Mantegazza Man96, Definition 3.1] then $V$ satisfies the hypotheses of Theorem 1 and, taking $C$ as in its conclusion, the second fundamental form of $V$ agrees almost everywhere with the second fundamental form induced by the members $M$ of $C$.

Evidently, Theorem 1 implies that the function mapping $\|V\|$ almost every $z$ onto the orthogonal projection of $\mathbf{R}^{n}$ onto the approximate $m$ dimensional tangent plane of $\|V\|$ at $z$ is $(\|V\|, m)$ approximately differentiable. If the first variation of $\|V\|$ satisfies the integrability condition $\left(H_{p}\right.$ ) below with sufficiently large exponent $p$ then this map is in fact differentiable in a stronger $\mathbf{L}_{2}\left(\|V\|, \operatorname{Hom}\left(\mathbf{R}^{n}, \mathbf{R}^{n}\right)\right)$ sense. Whenever $U$ is an open subset of $\mathbf{R}^{n}, V \in$ $\mathbf{I V}_{m}(U)$ and $1 \leq p \leq \infty$, the varifold $V$ is said to satisfy $\left(H_{p}\right)$ if and only if $\|\delta V\|$ is a Radon measure and, if $p>1$,

$$
\begin{aligned}
& (\delta V)(g)=-\int \mathbf{h}(V ; z) \bullet g(z) \mathrm{d}\|V\| z \quad \text { for } g \in \mathscr{D}\left(U, \mathbf{R}^{n}\right), \\
& \mathbf{h}(V ; \cdot) \in \mathbf{L}_{p}\left(\|V\|\left\llcorner K, \mathbf{R}^{n}\right) \quad \text { whenever } K \text { is a compact subset of } U .\right.
\end{aligned}
$$

Theorem 2 (see 4.2 and 4.5). Suppose $U$ is an open subset of $\mathbf{R}^{n}, 1 \leq p \leq \infty$, and $V \in \mathbf{I V}_{m}(U)$ satisfies $H_{p}$.

If either $m=1$ or $m=2$ and $p>1$ or $m>2$ and $p \geq 2 m /(m+2)$, then for $\|V\|$ almost all a

$$
f_{\mathbf{B}(a, r)}(\mid R(z)-R(a)-\langle R(a)(z-a), \text { ap } D R(a)\rangle|/| z-a \mid)^{2} \mathrm{~d}\|V\| z \rightarrow 0
$$

as $r \rightarrow 0+$ where $R(z)=\operatorname{Tan}^{m}(\|V\|, z)_{\natural} \in \operatorname{Hom}\left(\mathbf{R}^{n}, \mathbf{R}^{n}\right)$ and the approximate differential is taken with respect to $(\|V\|, m)$.

With the possible exception of the case $m=2$ this differentiability result is optimal with respect to the assumptions on $p$, i.e. whenever $m>2$ and $\frac{m p}{m-p}<2$ there exists an integral varifold satisfying $\left[H_{p}\right.$ not having the property in question, see 4.4

In previous work Schätzle established the following result in codimension one of the existence of submanifolds of class $\infty$ touching a given varifold, see Sch04, Proposition 4.1, Theorem 5.1] where it is phrased in terms of upper and lower height functions. 
Theorem (Schätzle Sch04]). Suppose $U$ is an open subset of $\mathbf{R}^{n}, p>m=$ $n-1, p \geq 2$, and $V \in \mathbf{I V}_{m}(U)$ satisfies $H_{p}$.

Then for $\|V\|$ almost all a there exists $0<r<\infty$ such that

$$
\mathbf{U}(a+v, r) \cap \operatorname{spt}\|V\|=\emptyset
$$

whenever $v \in \operatorname{Nor}^{m}(\|V\|, a)$ with $|v|=r$.

This is the key to showing that such a varifold satisfies the conclusion of Theorem 1, see Schätzle [Sch04, Theorem 6.1], and, in combination with previous results of the author in [Men09a, 3.7, 3.9], also that it satisfies the conclusion of Theorem 2. Evidently, see for example [Men09a, 1.2], Schätzle's Theorem does not extend to the case $p<m$. Also, the use of the theory of viscosity solutions for fully nonlinear equations, more precisely the results of Caffarelli Caf89 and Trudinger [Tru89], leads to the restriction to codimension one, i.e. $m=n-1$.

Therefore, in order to establish Theorem 1, a different method needs to be developed which is able to deal both with the low integrability of the generalised mean curvature and with higher codimension. The main independent result in this process is the following Theorem stated here in the case of Laplace's operator.

Theorem 3 (see 2.10). Suppose $U$ is an open subset of $\mathbf{R}^{m}, u: U \rightarrow \mathbf{R}^{n-m}$ is weakly differentiable, $j \in\{0,1\}, 1 \leq q<\infty$,

$$
h(a, r)=\inf \left\{\sum_{i=0}^{j} r^{i-m / q}\left|\mathbf{D}^{i}(u-v)\right|_{q ; a, r}: v \in \mathscr{E}\left(\mathbf{U}(a, r), \mathbf{R}^{n-m}\right), \operatorname{Lap} v=0\right\}
$$

whenever $a \in U, 0<r<\infty$ with $\mathbf{U}(a, r) \subset U$ and $A$ denotes the set of all $a \in U$ such that

$$
\limsup _{r \rightarrow 0+} r^{-2} h(a, r)<\infty .
$$

Then for $\mathscr{L}^{m}$ almost all $a \in A$ there exists a polynomial function $Q_{a}: \mathbf{R}^{m} \rightarrow$ $\mathbf{R}^{n-m}$ of degree at most 2 such that

$$
\lim _{r \rightarrow 0+} r^{-2} \sum_{i=0}^{j} r^{i-m / q}\left|\mathbf{D}^{i}\left(u-Q_{a}\right)\right|_{q ; a, r}=0 .
$$

Here the seminorms $|\cdot|_{q ; a, r}$ correspond to $\mathbf{L}_{q}\left(\mathscr{L}^{m}\llcorner\mathbf{U}(a, r))\right.$. The weaker statement which results when the condition Lap $v=0$ is replaced by $D^{2} v=0$ is contained in Calderón and Zygmund CZ61, Theorem 5] if $q>1$. However, the construction of affine comparison functions at a given point from information on the distributional Laplacian of $u$ may - for integral orders of differentiability - fail at individual points, see Men09b, 8.6]. This corresponds to the well known fact of the nonexistence of Schauder estimates for the Hölder exponent 1. In this respect the value of the current theorem stems from the fact that harmonic comparison functions are readily constructed independent of the order of differentiability considered, cp. 2.12. In fact, if $j=1, q>1$ and denoting by $T \in \mathscr{D}^{\prime}\left(U, \mathbf{R}^{n-m}\right)$ the distributional Laplacian of $u$ then

$$
\Gamma^{-1} h(a, r) \leq r^{1-m / q}|T|_{-1, q ; a, r} \leq \Gamma h(a, r)
$$

whenever $a \in U, 0<r<\infty, \mathbf{U}(a, r) \subset U$ and $u \mid \mathbf{U}(a, r) \in \mathbf{W}^{1, q}\left(\mathbf{U}(a, r), \mathbf{R}^{n-m}\right)$ where $\Gamma$ is a positive, finite number depending only on $n$ and $q$ and $|\cdot|_{-1, q ; a, r}$ 
denotes the seminorm corresponding to $\left(\mathbf{W}_{0}^{1, q /(q-1)}\left(\mathbf{U}(a, r), \mathbf{R}^{n-m}\right)\right)^{*}$. In particular, if $T$ is representable by integration and $q<m /(m-1)$ if $m>1$ then one verifies $\mathscr{L}^{m}(U \sim A)=0$. An extensive study of both integral and nonintegral orders of differentiability for solutions of linear elliptic partial differential equations in nondivergence form can be found in Calderón and Zygmund [CZ61].

In passing to divergence form equations, one is naturally lead to consider the related problem for distributions:

Theorem 4 (see 2.12 and A.3). Suppose $U$ is an open subset of $\mathbf{R}^{m}, 1 \leq q<\infty$, $T \in \mathscr{D}^{\prime}\left(U, \mathbf{R}^{n-m}\right)$ and $A$ denotes the set of all $a \in U$ such that

$$
\limsup _{r \rightarrow 0+} r^{-1-m / q}|T|_{-1, q ; a, r}<\infty .
$$

Then for $\mathscr{L}^{m}$ almost every a $\in A$ there exists a unique constant distribution $T_{a} \in \mathscr{D}^{\prime}\left(U, \mathbf{R}^{n-m}\right)$ such that

$$
\lim _{r \rightarrow 0+} r^{-1-m / q}\left|T-T_{a}\right|_{-1, q ; a, r}=0 .
$$

This may be seen as a Lebesgue point theorem for distributions. In case $q>1$, it is in fact a corollary to Theorem 3 obtainable by representing $T$ locally as distributional Laplacian of some function $u$. In contrast, the case $q=1$ is independent from the other results of the present paper.

Finally, it should be noted that the proof of Theorem 3 only relies on a priori estimates in Lebesgue spaces, i.e. "L $\mathbf{L}_{p}$ theory", which are known to hold for a much wider class of linear equations, see Agmon, Douglis and Nirenberg ADN59, ADN64].

Outline of the proofs To prove Theorem 3 , one considers the subsets of $A_{k}$ of $A$ of all $a \in A$ such $h(a, r) \leq k r^{2}$ whenever $0<r<1 / k$. Denoting by $v_{a, r}$ : $\mathbf{U}(a, r) \rightarrow \mathbf{R}^{n-m}$ harmonic functions essentially realising the infimum in the definition of $h$, one then uses the partition of unity with estimates from Fed69, 3.1.13] together with well known a priori estimates for the Laplace operator to construct functions $v_{k}: \mathbf{R}^{m} \rightarrow \mathbf{R}^{n-m}$ with the following properties, see 2.8

(1) There holds

$$
\sum_{i=0}^{j} r^{i-m / q}\left|\mathbf{D}^{i}\left(v_{k}-u\right)\right|_{q ; a, r} \leq \Gamma k r^{2}
$$

for $a \in A_{k}$ and $0<r<(36 k)^{-1}$ and $\Gamma$ a positive, finite number depending only on $n$ and $q$, in particular $v_{k}(x)=u(x)$ for $\mathscr{L}^{m}$ almost all $x \in A_{k}$.

(2) The distributional Laplacian of $v_{k}$ is represented by a function locally in $\mathbf{L}_{\infty}\left(\mathscr{L}^{m}, \mathbf{R}^{n-m}\right)$.

Then clearly $v_{k}$ locally belongs to $\mathbf{W}^{2, q}\left(\mathbf{R}^{m}, \mathbf{R}^{n-m}\right)$ for $1 \leq q<\infty$ and the conclusion of Theorem 3 follows from by now classical differentiability results for functions in Sobolev spaces which where also obtained by Calderón and Zygmund in CZ61. An important feature of this proof is that it is readily adapted to the case where the Laplace operator is replaced by the Euler Lagrange differential operator $L_{F}$ corresponding to an integrand $F: \operatorname{Hom}\left(\mathbf{R}^{m}, \mathbf{R}^{n-m}\right) \rightarrow \mathbf{R}$ of class 2 sufficiently close to the Dirichlet integrand, i.e. Lip $D^{2} F<\infty$ and

$$
\left|\left\langle\left(\tau_{1}, \tau_{2}\right), D^{2} F(\sigma)\right\rangle-\tau_{1} \bullet \tau_{2}\right| \leq \varepsilon \quad \text { for } \sigma, \tau_{1}, \tau_{2} \in \operatorname{Hom}\left(\mathbf{R}^{m}, \mathbf{R}^{n-m}\right)
$$


with suitable number $\varepsilon$.

Next, it will explained how this result on a rather restricted class of differential operators can be used to treat the general case. For this purpose let $U$ be an open subset of $\mathbf{R}^{n}$ and let $V \in \mathbf{I V}_{m}(U)$ be such that $\|\delta V\|$ is a Radon measure. Comparing the behaviour of $V$ near certain "good" points to the behaviour of harmonic functions, a procedure developed by De Giorgi in DG61] and Almgren in Alm68], one proves the tilt decay estimate

$$
\limsup _{r \rightarrow 0+} r^{-\tau-m / 2}\left(\int_{\mathbf{U}(a, r) \times \mathbf{G}(n, m)}\left|S_{\natural}-T_{\natural}\right|^{2} \mathrm{~d} V(z, S)\right)^{1 / 2}<\infty
$$

for $V$ almost all $(a, T)$ where $0<\tau<1$ if $m \in\{1,2\}$ and $\tau=\frac{m}{2(m-1)}<1$ if $m>2$. This has been done by the author in [Men09b, 8.6] extending results of Brakke [Bra78, 5.7,5] who proved the case $\tau=1 / 2$ with " $<\infty$ " replaced by $=0$ which is sufficient for the proof of all Theorems stated in the Introduction. As the order of differentiability considered is nonintegral, i.e. $0<\tau<1$, the argument applies, in contrast to those of the present paper, in a direct way to all points satisfying a simple set of conditions, see [Men09b, 8.3].

The principal idea to prove Theorem 1 is now to use the tilt decay estimate, to construct a sequence functions $g_{i}: \mathbf{R}^{m} \rightarrow \mathbf{R}^{n-m}, \mathscr{L}^{m}$ measurable sets $K_{i} \subset \mathbf{R}^{m}$ and distributions $T_{i} \in \mathscr{D}^{\prime}\left(\mathbf{R}^{m}, \mathbf{R}^{n-m}\right)$ with the following properties:

(1) The varifold is covered by suitably rotated graphs of the $g_{i} \mid K_{i}$.

(2) The distribution $T_{i}$ corresponds to the Euler Lagrange differential operator associated to the nonparametric area integrand $\Phi$ applied to $g_{i}$.

(3) There holds

$$
\lim _{r \rightarrow 0+} r^{-1-m} \int_{\mathbf{U}(x, r)}\left|D g_{i}(\zeta)-D g_{i}(x)\right|^{2} \mathrm{~d} \mathscr{L}^{m} \zeta=0 \quad \text { whenever } x \in K_{i} .
$$

(4) The Lipschitz constant of the $g_{i}$ is small.

(5) The distributions $T_{i}$ satisfy the conclusion of Theorem 4 with $q=1$ and $A$ replaced by $K_{i}$ with constant distribution given by the generalised mean curvature of the varifold.

Condition (4) is the minimum condition needed to be able to replace $\Phi$ with some integrand $F$ of the type discussed before in the definition of $T_{i}$ without changing it, see 2.20 The basis for the construction of $g_{i}, K_{i}$, and $T_{i}$ is an approximation by $\mathbf{Q}_{Q}\left(\mathbf{R}^{n-m}\right)$ valued functions where the space $\mathbf{Q}_{Q}\left(\mathbf{R}^{n-m}\right)$ is isometric to the $Q$ fold product of $\mathbf{R}^{n-m}$ divided by the action of group of permutations of $\{1, \ldots, Q\}$. Here the version of the author in Men09b, 4.8] is employed which contains some estimates designed for the current applications and was obtained by combining and extending similar constructions of Almgren in Alm00, $\S 3]$ and Brakke in [Bra78, 5.4]. This yields Lipschitzian functions $f_{i}: K_{i} \rightarrow \mathbf{Q}_{Q_{i}}\left(\mathbf{R}^{n-m}\right)$ with small Lipschitz constant for suitable positive integers $Q_{i}$. Denoting the "centre" of $S \in \mathbf{Q}_{Q}\left(\mathbf{R}^{n-m}\right)$ by $\boldsymbol{\eta}_{Q}(S)=Q^{-1} \sum_{j=1}^{Q} y_{j}$ whenever $y_{1}, \ldots, y_{Q} \in$ $\mathbf{R}^{n-m}$ correspond to $S$, the functions $g_{i}$ are then constructed in 3.3 as extensions of $\boldsymbol{\eta}_{Q_{i}} \circ f_{i}$. In this process the conditions (3) and (5) are ultimately consequences of the tilt decay estimate. 
The final step in the proof of Theorem 1 is now to construct for fixed $i$ and $x \in K_{i}$ comparison functions $v_{r} \in \mathbf{W}^{1,2}\left(\mathbf{U}(x, r), \mathbf{R}^{n-m}\right)$ with $L_{F}\left(v_{r}\right)=0$ for $0<r<\infty$ and estimating $g_{i}-v_{r}$ in $\mathbf{U}(x, r)$, see 2.13 2.17. The natural choice is to take $v_{r}$ as solution of the Dirichlet problem with boundary values given by $g_{i}$. If $q$ in (5) would satisfy $q>1$ this would immediately yield an estimate of $g_{i}-v_{r}$ in $\mathbf{W}^{1, q}\left(\mathbf{U}(x, r), \mathbf{R}^{n-m}\right)$. In case $q=1$ the estimate needs to be obtained differently, namely, linearising $F$ and estimating the remaining terms with the help of condition (3), one obtains an estimate in $\mathbf{L}_{1}\left(\mathbf{U}(x, r), \mathbf{R}^{n-m}\right)$ instead, see 2.15. Then the extended version of Theorem 3 with $L_{F}$ replacing Lap, see 2.10, implies the first part of Theorem 11 Recalling condition (5), the second part is derived similarly by using functions $w_{r} \in \mathbf{W}^{1,2}\left(\mathbf{U}(x, r), \mathbf{R}^{n-m}\right)$ with $L_{F}\left(w_{r}\right)=\left(T_{i}\right)_{x}$ where $\left(T_{i}\right)_{x}$ is the constant distribution corresponding to $T_{i}$ at $x$ as in Theorem 4 .

Organisation of paper In Section 1 the Notation is fixed. Section 2 contains all results which can be phrased solely in terms of elliptic partial differential equations and distributions, in particular Theorem [3] and the case $q>1$ of Theorem 4. Section 3 is devoted to the proof of Theorem 1 whereas Section 4 contains Theorem 2, Finally, Appendix A gives the proof of the case $q=1$ of Theorem 4 .

Acknowledgements The author offers his thanks to his $\mathrm{PhD}$ advisor Professor Dr. Reiner Schätzle who lead him towards the study of this problem. The author also thanks Professor Dr. Tom Ilmanen for several related discussions.

\section{Notation}

The notation from Federer [Fed69] and Allard All72] is used with some modifications and additions described in Men09b, $\S 1, \S 2]$. Additionally, whenever $M$ is a submanifold of $\mathbf{R}^{n}$ of class 2 the mean curvature of $M$ at $z \in M$ is denoted by $\mathbf{h}(M ; z)$, cp. Allard All72, 2.5 (2)]. And if $U$ is an open subset of $\mathbf{R}^{m}$ and $Y$ is a Banach space then $T$ is called a constant distribution in $U$ of type $Y$ if and only if for some $\alpha \in Y^{*}$ there holds $T(\theta)=\int_{U} \alpha \circ \theta \mathrm{d} \mathscr{L}^{m}$ for $\theta \in \mathscr{D}(U, Y)$. Moreover, a subset of a topological space is called universally measurable if and only if it is measurable with respect to every Borel measure on that space.

The reader might want to recall the following maybe less commonly used symbols either taken from [Fed69, 2.2.6, 2.8.1, 1.10.1] or introduced in Men09b, $\S 1]: \mathscr{P}$ denoting the positive integers, $\mathbf{U}(a, r)$ and $\mathbf{B}(a, r)$ denoting respectively the open and closed ball with centre $a$ and radius $r, \bigodot^{i}(V, W)$ and $\bigodot^{i} V$ denoting the vector space of all $i$ linear symmetric functions (forms) mapping $V^{i}$ into $W$ and $\mathbf{R}$ respectively and the seminorms

$$
\begin{aligned}
|f|_{p ; a, r} & =\left(\int_{\mathbf{U}(a, r)}|f|^{p} \mathrm{~d} \mathscr{L}^{m}\right)^{1 / p} \quad \text { if } p<\infty, \\
|f|_{\infty ; a, r} & =\inf \left\{t: \mathscr{L}^{m}(\mathbf{U}(a, r) \cap\{x:|f(x)|>t\})=0\right\}, \\
|T|_{i, q ; a, r} & =\sup \left\{T(\theta): \theta \in \mathscr{D}\left(U, \mathbf{R}^{n-m}\right), \operatorname{spt} \theta \subset \mathbf{U}(a, r),\left|D^{-i} \theta\right|_{p ; a, r} \leq 1\right\}
\end{aligned}
$$

whenever $m, n \in \mathscr{P}, m<n, U$ is an open subset of $\mathbf{R}^{m}, a \in \mathbf{R}^{m}, 0<r<\infty$ with $\mathbf{U}(a, r) \subset U, f$ is an $\mathscr{L}^{m}\llcorner\mathbf{U}(a, r)$ measurable function with values in a 
Hilbert space, $1 \leq p \leq \infty, 1 \leq q \leq \infty$ with $1 / p+1 / q=1, i$ is a negative integer, and $T \in \mathscr{D}^{\prime}\left(U, \mathbf{R}^{m}\right)$.

\section{A criterion for second order differentiability in Lebesgue spaces}

The purpose of this section is to prove 2.10 which contains Theorem 3 of the Introduction and to provide the preparations necessary for its application in Section 3

First, in 2.1 the situation studied is described. Then, for the convenience of the reader, in 2.2 2.7 adaptions and applications of standard theory are carried out. The main ingredient in the proof of 2.10 is contained in 2.8 The part $q>1$ of Theorem 4 is provided in 2.12 Finally, in 2.13 2.17 it is shown how a certain nonintegral differentiability condition on the solution $u$ allows to treat the case where estimates for $L_{F}(u)$, see 2.1, are only available in $|\cdot|_{-1,1 ; a, r}$.

2.1. Suppose $m, n \in \mathscr{P}, m<n$,

$$
e_{1}, \ldots, e_{m} \text { and } X_{1}, \ldots, X_{m}
$$

are dual orthonormal bases of $\mathbf{R}^{m}$ and $\bigodot^{1} \mathbf{R}^{m}$ and

$$
v_{1}, \ldots, v_{n-m} \text { and } Y_{1}, \ldots, Y_{n-m}
$$

are dual orthonormal bases of $\mathbf{R}^{n-m}$ and $\bigodot^{1} \mathbf{R}^{n-m}$. The norm $\|\Psi\|$ denotes for any $\Psi \in \bigodot^{2} \operatorname{Hom}\left(\mathbf{R}^{m}, \mathbf{R}^{n-m}\right)$ the smallest nonnegative number $M$ such that

$$
\Psi(\sigma, \tau) \leq M|\sigma||\tau| \text { for } \sigma, \tau \in \operatorname{Hom}\left(\mathbf{R}^{m}, \mathbf{R}^{n-m}\right) .
$$

The expression $\Psi(\sigma, \tau)$ for $\sigma, \tau \in \operatorname{Hom}\left(\mathbf{R}^{m}, \mathbf{R}^{n-m}\right)$ will be denoted alternately by $\langle(\sigma, \tau), \Psi\rangle$ and, using $\odot$ to denote multiplication in $\bigodot_{*} \operatorname{Hom}\left(\mathbf{R}^{m}, \mathbf{R}^{n-m}\right)$, see [Fed69, 1.9.1], also by $\langle\sigma \odot \tau, \Psi\rangle$. It equals

$$
\sum_{i=1}^{m} \sum_{j=1}^{n-m} \sum_{k=1}^{m} \sum_{l=1}^{n-m} \Psi_{i, j ; k, l}\left\langle\sigma\left(e_{i}\right), Y_{j}\right\rangle\left\langle\tau\left(e_{k}\right), Y_{l}\right\rangle
$$

where $\Psi_{i, j ; k, l}=\Psi\left(X_{i} v_{j}, X_{k} v_{l}\right)$ and $X v$ maps $x \in \mathbf{R}^{m}$ onto $X(x) v \in \mathbf{R}^{n-m}$ whenever $X \in \odot^{1} \mathbf{R}^{m}$ and $v \in \mathbf{R}^{n-m}$.

Let $\Upsilon \in \bigodot^{2} \operatorname{Hom}\left(\mathbf{R}^{m}, \mathbf{R}^{n-m}\right)$ be defined by

$$
\Upsilon(\sigma, \tau)=\sigma \bullet \tau \quad \text { for } \sigma, \tau \in \operatorname{Hom}\left(\mathbf{R}^{m}, \mathbf{R}^{n-m}\right),
$$

and suppose $F: \operatorname{Hom}\left(\mathbf{R}^{m}, \mathbf{R}^{n-m}\right) \rightarrow \mathbf{R}$ is of class $2,0 \leq \varepsilon<\infty$, and

$$
\left\|D^{2} F(\sigma)-\Upsilon\right\| \leq \varepsilon \quad \text { whenever } \sigma \in \operatorname{Hom}\left(\mathbf{R}^{m}, \mathbf{R}^{n-m}\right) .
$$

The quantity Lip $D^{2} F$ will be computed with respect to $|\cdot|$ on $\operatorname{Hom}\left(\mathbf{R}^{m}, \mathbf{R}^{n-m}\right)$ and $\|\cdot\|$ on $\bigodot^{2} \operatorname{Hom}\left(\mathbf{R}^{m}, \mathbf{R}^{n-m}\right)$.

To each such $F$ there corresponds the Euler Lagrange differential operator $L_{F}$ which associates to every $u \in \mathbf{W}^{1,1}\left(U, \mathbf{R}^{n-m}\right)$ for some open subset $U$ of $\mathbf{R}^{m}$ a distribution $L_{F}(u)$ in $\mathscr{D}^{\prime}\left(U, \mathbf{R}^{n-m}\right)$ defined by

$$
L_{F}(u)(\theta)=-\int_{U}\langle D \theta(x), D F(\mathbf{D} u(x))\rangle \mathrm{d} \mathscr{L}^{m} x \quad \text { for } \theta \in \mathscr{D}\left(U, \mathbf{R}^{n-m}\right) .
$$


There also occurs the linear function $C_{F}(\sigma): \bigodot^{2}\left(\mathbf{R}^{m}, \mathbf{R}^{n-m}\right) \rightarrow \mathbf{R}^{n-m}$ which for $\sigma \in \operatorname{Hom}\left(\mathbf{R}^{m}, \mathbf{R}^{n-m}\right)$ is given by

$$
\left\langle\phi, C_{F}(\sigma)\right\rangle=\sum_{i=1}^{m} \sum_{j=1}^{n-m} \sum_{k=1}^{m} \sum_{l=1}^{n-m}\left\langle\left(X_{i} v_{j}, X_{k} v_{l}\right), D^{2} F(\sigma)\right\rangle\left\langle\phi\left(e_{i}, e_{k}\right), Y_{j}\right\rangle v_{l}
$$

whenever $\phi \in \bigodot^{2}\left(\mathbf{R}^{m}, \mathbf{R}^{n-m}\right)$. The function $C_{F}(\sigma)$ is uniquely determined by $D^{2} F(\sigma)$, see Fed69, 5.2.11]. One obtains by partial integration for $u \in$ $\mathbf{W}^{2,1}\left(U, \mathbf{R}^{n-m}\right), \theta \in \mathscr{D}\left(U, \mathbf{R}^{n-m}\right)$

$$
L_{F}(u)(\theta)=\int_{U} \theta(x) \bullet\left\langle\mathbf{D}^{2} u(x), C_{F}(\mathbf{D} u(x))\right\rangle \mathrm{d} \mathscr{L}^{m} x .
$$

Sometimes also $S: \bigodot^{2}\left(\mathbf{R}^{m}, \mathbf{R}^{n-m}\right) \rightarrow \mathbf{R}^{n-m}$ corresponding to the Dirichlet integrand, i.e. $F(\sigma)=|\sigma|^{2} / 2$ for $\sigma \in \operatorname{Hom}\left(\mathbf{R}^{m}, \mathbf{R}^{m}\right)$, (and therefore to $\Upsilon$ ) will be used. Note $\langle\phi, S\rangle=\sum_{i=1}^{m} \phi\left(e_{i}, e_{i}\right)$ whenever $\phi \in \bigodot^{2}\left(\mathbf{R}^{m}, \mathbf{R}^{n-m}\right)$. One may check that with $\kappa=2^{1 / 2} m(n-m)$

$$
\begin{gathered}
\left|C_{F}(\sigma)\right| \leq \kappa\left\|D^{2} F(\sigma)\right\|, \quad\left|C_{F}(\sigma)-S\right| \leq \kappa \varepsilon, \\
\left|C_{F}(\sigma)-C_{F}(\tau)\right| \leq \kappa\left\|D^{2} F(\sigma)-D^{2} F(\tau)\right\|
\end{gathered}
$$

for $\sigma, \tau \in \operatorname{Hom}\left(\mathbf{R}^{m}, \mathbf{R}^{n-m}\right)$ where $|\cdot|$ denotes the norm associated to the inner product on Hom $\left(\bigodot^{2}\left(\mathbf{R}^{m}, \mathbf{R}^{n-m}\right), \mathbf{R}^{n-m}\right)$, see [Fed69, 1.7.9, 1.10.6].

2.2 Theorem. Suppose $n \in \mathscr{P}$ and $1<p<\infty$.

Then there exist positive, finite numbers $\varepsilon$ and $\Gamma$ with the following property.

If $n>m \in \mathscr{P}$, $\Upsilon$ is as in 2.1, $a \in \mathbf{R}^{m}, 0<r<\infty$,

$$
\begin{gathered}
A: \mathbf{U}(a, r) \rightarrow \bigodot^{2} \operatorname{Hom}\left(\mathbf{R}^{m}, \mathbf{R}^{n-m}\right) \text { is } \mathscr{L}^{m}\llcorner\mathbf{U}(a, r) \text { measurable, } \\
\|A(x)-\Upsilon\| \leq \varepsilon \quad \text { whenever } x \in \mathbf{U}(a, r),
\end{gathered}
$$

then for every $T \in \mathscr{D}^{\prime}\left(\mathbf{U}(a, r), \mathbf{R}^{n-m}\right)$ with $|T|_{-1, p ; a, r}<\infty$ there exists an $\mathscr{L}^{m}\left\llcorner\mathbf{U}(a, r)\right.$ almost unique $u \in \mathbf{W}_{0}^{1, p}\left(\mathbf{U}(a, r), \mathbf{R}^{n-m}\right)$ such that

$$
-\int_{\mathbf{U}(a, r)}\langle D \theta(x) \odot \mathbf{D} u(x), A(x)\rangle \mathrm{d} \mathscr{L}^{m} x=T(\theta) \quad \text { for } \theta \in \mathscr{D}\left(\mathbf{U}(a, r), \mathbf{R}^{n-m}\right) .
$$

Moreover, whenever $u$ and $T$ are related as above there holds

$$
|\mathbf{D} u|_{p ; a, r} \leq \Gamma|T|_{-1, p ; a, r} .
$$

Proof. By the Neumann series (cf. Fed69, 3.1.11]) it is enough to consider the case $\varepsilon=0$. Note also that there exists $g \in \mathbf{L}_{p}\left(\mathscr{L}^{m}\left\llcorner\mathbf{U}(a, r), \operatorname{Hom}\left(\mathbf{R}^{m}, \mathbf{R}^{n-m}\right)\right)\right.$ with $T(\theta)=-\int_{\mathbf{U}(a, r)} g \bullet D \theta \mathrm{d} \mathscr{L}^{m}$ for $\theta \in \mathscr{D}\left(\mathbf{U}(a, r), \mathbf{R}^{n-m}\right)$ and $|T|_{-1, p ; a, r}=$ $|g|_{p ; a, r}$ by Hahn Banach's theorem.

The conclusion then follows from [Giu03, Theorem 10.15] in case $p \geq 2$ to which the case $p<2$ reduces by use of a duality argument.

2.3 Theorem. Suppose $n \in \mathscr{P}, 1<q<\infty$, and $1<p<\infty$.

Then there exists a positive, finite number $\varepsilon$ with the following property.

If $n>m \in \mathscr{P}$, $\Upsilon$ is as in 2.1, $a \in \mathbf{R}^{m}, 0<r<\infty$,

$$
\begin{gathered}
A: \mathbf{U}(a, r) \rightarrow \bigodot^{2} \operatorname{Hom}\left(\mathbf{R}^{m}, \mathbf{R}^{n-m}\right) \text { is } \mathscr{L}^{m}\llcorner\mathbf{U}(a, r) \text { measurable, } \\
\|A(x)-\Upsilon\| \leq \varepsilon \quad \text { whenever } x \in \mathbf{U}(a, r),
\end{gathered}
$$


and $u \in \mathbf{W}^{1, q}\left(\mathbf{U}(a, r), \mathbf{R}^{n-m}\right), T \in \mathscr{D}^{\prime}\left(\mathbf{U}(a, r), \mathbf{R}^{n-m}\right)$ satisfy

$$
-\int_{\mathbf{U}(a, r)}\langle D \theta(x) \odot \mathbf{D} u(x), A(x)\rangle \mathrm{d} \mathscr{L}^{m} x=T(\theta) \quad \text { for } \theta \in \mathscr{D}\left(\mathbf{U}(a, r), \mathbf{R}^{n-m}\right),
$$

then

$$
|\mathbf{D} u|_{p ; a, r / 2} \leq \Gamma\left(r^{-m-1+m / p}|u|_{1 ; a, r}+|T|_{-1, p ; a, r}\right)
$$

where $\Gamma$ is a positive, finite number depending only on $n$ and $p$.

Proof. Let $0<\delta \leq 1$, suppose $n, q, p, m, \Upsilon, a, r, A, u$, and $T$ satisfy the hypotheses in the body of the theorem with $\varepsilon$ replaced by $\delta$ and assume $q \leq p$. It will be shown that $u$ satisfies the estimate in the conclusion of the theorem provided $\delta$ is suitably small.

The problem will be reduced.

First, to the case $p=q$ by constructing as solutions of approximating Dirichlet problems by use of 2.2 a sequence of functions $u_{i} \in \mathbf{W}^{1, p}\left(\mathbf{U}(a, r), \mathbf{R}^{n-m}\right)$ such that $u_{i} \rightarrow u$ in $\mathbf{W}^{1, q}\left(\mathbf{U}(a, r), \mathbf{R}^{n-m}\right)$ as $i \rightarrow \infty$ and for $i \in \mathscr{P}$

$$
-\int_{\mathbf{U}(a, r)}\left\langle D \theta(x) \odot \mathbf{D} u_{i}(x), A(x)\right\rangle \mathrm{d} \mathscr{L}^{m} x=T(\theta) \quad \text { for } \theta \in \mathscr{D}\left(\mathbf{U}(a, r), \mathbf{R}^{n-m}\right)
$$

provided $\delta \leq \inf \left\{q_{2.2}(n, p), q 2.2(n, q)\right\}$.

Secondly, to the case $p=q$ and $\delta=0$ by considering Simon's absorption lemma in Sim97, p. 398].

Thirdly, to the case $p=q, \delta=0$ and $T=0$ by use of 2.2 and Poincaré's inequality.

Finally, the remaining case follows by convolution from GT01, Theorems $2.8,2.10]$.

2.4 Theorem. Suppose $n \in \mathscr{P}$ and $1<p<\infty$.

Then there exists a positive, finite number $\varepsilon$ with the following property.

If $n>m \in \mathscr{P}, S$ is as in 2.1, $a \in \mathbf{R}^{m}, 0<r<\infty$,

$$
\begin{gathered}
B: \mathbf{U}(a, r) \rightarrow \operatorname{Hom}\left(\bigodot^{2}\left(\mathbf{R}^{m}, \mathbf{R}^{n-m}\right), \mathbf{R}^{n-m}\right) \text { is } \mathscr{L}^{m}\llcorner\mathbf{U}(a, r) \text { measurable }, \\
|B(x)-S| \leq \varepsilon \quad \text { whenever } x \in \mathbf{U}(a, r),
\end{gathered}
$$

and $u \in \mathbf{W}^{2, p}\left(\mathbf{U}(a, r), \mathbf{R}^{n-m}\right), f \in \mathbf{L}_{p}\left(\mathscr{L}^{m}\left\llcorner\mathbf{U}(a, r), \mathbf{R}^{n-m}\right)\right.$ satisfy

$$
\left\langle\mathbf{D}^{2} u(x), B(x)\right\rangle=f(x) \quad \text { for } \mathscr{L}^{m} \text { almost all } x \in \mathbf{U}(a, r),
$$

then

$$
\left|\mathbf{D}^{2} u\right|_{p ; a, r / 2} \leq \Gamma\left(r^{-2-m+m / p}|u|_{1 ; a, r}+|f|_{p ; a, r}\right)
$$

where $\Gamma$ is a positive, finite number depending only on $n$ and $p$.

Proof. From [GT01, Theorem 7.22] and Ehring's lemma, see e.g. Wlo87, Theorem I.7.3], it follows that for every $0<\kappa<\infty$ there exists a positive, finite number $\Delta$ depending only on $n, p$, and $\kappa$ such that

$$
r^{-2-m / p}|v|_{p ; a, r} \leq \kappa r^{-m / p}\left|\mathbf{D}^{2} v\right|_{p ; a, r}+\Delta r^{-2-m}|v|_{1 ; a, r}
$$

for $v \in \mathbf{W}^{2, p}\left(\mathbf{U}(a, r), \mathbf{R}^{n-m}\right)$.

Now, one may readily use GT01, Theorem 9.11] in conjunction with the absorption lemma in Simon Sim97, p. 398] to obtain the conclusion. 
2.5 Lemma. Suppose $n \in \mathscr{P}, 1<q<\infty$, and $1<p<\infty$.

Then there exists a positive, finite number $\varepsilon$ with the following property.

If $F$ is related to $\varepsilon$ as in 2.1, $a \in \mathbf{R}^{m}, 0<r<\infty, u \in \mathbf{W}^{1, q}\left(\mathbf{U}(a, r), \mathbf{R}^{n-m}\right)$, and $f \in \mathbf{L}_{p}\left(\mathscr{L}^{m}\left\llcorner\mathbf{U}(a, r), \mathbf{R}^{n-m}\right)\right.$ satisfy

$$
L_{F}(u)(\theta)=\int_{\mathbf{U}(a, r)} \theta(x) \bullet f(x) \mathrm{d} \mathscr{L}^{m} x \quad \text { whenever } \theta \in \mathscr{D}\left(\mathbf{U}(a, r), \mathbf{R}^{n-m}\right),
$$

then $u$ is twice weakly differentiable and for every affine function $P: \mathbf{R}^{m} \rightarrow$ $\mathbf{R}^{n-m}$ there holds

$$
\left|\mathbf{D}^{2} u\right|_{p ; a, r / 2} \leq \Gamma\left(r^{-2-m+m / p}|u-P|_{1 ; a, r}+|f|_{p ; a, r}\right)
$$

where $\Gamma$ is a positive, finite number depending only on $n$ and $p$.

Proof. Let $\varepsilon=\varepsilon_{2.3}(n, q, p)$ and suppose $F, a, r, u, f$, and $P$ satisfy the hypotheses in body of the lemma.

Let $v=u-P, i \in\{1, \ldots, m\}$ and define for $0<h<r, x \in \mathbf{U}(a, r-h)$

$$
\begin{gathered}
u_{h}(x)=h^{-1}\left(u\left(x+h e_{i}\right)-u(x)\right), \quad v_{h}(x)=h^{-1}\left(v\left(x+h e_{i}\right)-v(x)\right), \\
A_{h}(x)=\int_{0}^{1} D^{2} F\left(t \mathbf{D} u\left(x+h e_{i}\right)+(1-t) \mathbf{D} u(x)\right) \mathrm{d} \mathscr{L}^{1} t,
\end{gathered}
$$

and let $S_{h} \in \mathscr{D}^{\prime}\left(\mathbf{U}(a, r-h), \mathbf{R}^{n-m}\right)$ be characterised by

$$
S_{h}(\theta \mid \mathbf{U}(a, r-h))=h^{-1} \int_{\mathbf{U}(a, r)}\left(\theta\left(x-h e_{i}\right)-\theta(x)\right) \bullet f(x) \mathrm{d} \mathscr{L}^{m} x
$$

whenever $\theta \in \mathscr{D}\left(\mathbf{R}^{m}, \mathbf{R}^{n-m}\right)$ with $\operatorname{spt} \theta \subset \mathbf{U}(a, r-h)$. One readily verifies, noting $\mathbf{D} u_{h}=\mathbf{D} v_{h}$,

$$
-\int_{\mathbf{U}(a, r-h)}\left\langle D \theta(x) \odot \mathbf{D} v_{h}(x), A_{h}(x)\right\rangle \mathrm{d} \mathscr{L}^{m} x=S_{h}(\theta)
$$

for $\theta \in \mathscr{D}\left(\mathbf{U}(a, r-h), \mathbf{R}^{n-m}\right)$. Hence, by 2.3 ,

$$
\left|\mathbf{D} v_{h}\right|_{p ; a,(r-h) / 2} \leq \Delta\left((r-h)^{-1-m+m / p}\left|v_{h}\right|_{1 ; a, r-h}+\left|S_{h}\right|_{-1, p ; a, r-h}\right)
$$

where $\Delta=\Gamma_{2.3}(n, p)$. Since $\left|v_{h}\right|_{1 ; a, r-h} \leq|\mathbf{D} v|_{1 ; a, r}$ and $\left|S_{h}\right|_{-1, p ; a, r-h} \leq$ $|f|_{p ; a, r}$, taking the limit $h \rightarrow 0+$ one infers that $v$, hence $u$, is twice weakly differentiable and satisfies the desired estimate, using Simon's absorption lemma [Sim97, p. 398] as before.

2.6 Remark. In general, even if $\operatorname{Lip} u \leq L<\infty$ and $P=0$ the condition involving $\varepsilon$ cannot be replaced by some uniform strong ellipticity condition on $D^{2} F(\sigma)$ for $\sigma \in \operatorname{Hom}\left(\mathbf{R}^{m}, \mathbf{R}^{n-m}\right)$ with $\|\sigma\| \leq L$ as may be seen from the example of Lawson and Osserman in [LO77, Theorem 7.1].

2.7 Lemma. Suppose $n \in \mathscr{P}$, and $1<q \leq p<\infty$.

Then there exists a positive, finite number $\varepsilon$ with the following property.

If $n>m \in \mathscr{P}, F$ is related to $\varepsilon$ as in 2.1, Lip $D^{2} F<\infty, a \in \mathbf{R}^{m}, 0<r<$ $\infty$, and $u_{i} \in \mathbf{W}^{1, q}\left(\mathbf{U}(a, r), \mathbf{R}^{n-m}\right)$ with $i \in\{1,2\}$ satisfy $L_{F}\left(u_{i}\right)=0$, then $u_{i}$ are twice weakly differentiable and for every affine function $P: \mathbf{R}^{m} \rightarrow \mathbf{R}^{n-m}$ there holds

$$
\begin{aligned}
r^{-m / p+1} \mid \mathbf{D}^{2}\left(u_{2}-\right. & \left.u_{1}\right)\left.\right|_{p ; a, r / 2} \leq \Gamma\left(r^{-m-1}\left|u_{2}-u_{1}\right|_{1 ; a, r}\right. \\
& \left.+\left(r^{-m-1}\left|u_{1}-P\right|_{1 ; a, r}\right) \operatorname{Lip}\left(D^{2} F\right)\left(r^{-m-1}\left|u_{2}-u_{1}\right|_{1 ; a, r}\right)\right)
\end{aligned}
$$

where $\Gamma$ is a positive, finite number depending only on $n$ and $p$. 
Proof. Using an elementary covering argument, it is enough to prove the assertion with $\left|\mathbf{D}^{2}\left(u_{2}-u_{1}\right)\right|_{p ; a, r / 2}$ replaced by $\left|\mathbf{D}^{2}\left(u_{2}-u_{1}\right)\right|_{p ; a, r / 4}$. For this purpose let $\kappa=2^{1 / 2} n^{2}$

$$
\begin{gathered}
\varepsilon=\inf \left\{q_{2.5}(n, q, 2 p), q_{2.4}(n, p) / \kappa, q 2.3(n, q, 2 p)\right\}, \quad \Delta_{1}=\Gamma[2.5(n, 2 p), \\
\Delta_{2}=\Gamma_{2.4}(n, p), \quad \Delta_{3}=\Gamma_{[2.3}(n, 2 p), \quad \Gamma=\Delta_{2} \sup \left\{2^{1+n}, \kappa \Delta_{1} \Delta_{3}\right\} .
\end{gathered}
$$

Suppose $F, a, r$, and $u_{i}$ satisfy the hypotheses with $\varepsilon$ and that $P: \mathbf{R}^{m} \rightarrow \mathbf{R}^{n-m}$ is an affine function. In order to show that they satisfy the modified conclusions with $\Gamma$, it will be assumed $a=0$ and $r=1$. Abbreviate $\Lambda=\operatorname{Lip} D^{2} F$.

By 2.5 the functions $u_{i}$ are twice weakly differentiable with

$$
\left|\mathbf{D}^{2} u_{i}\right|_{2 p ; 0,1 / 2} \leq \Delta_{1}\left|u_{i}-P\right|_{1 ; 0,1} \quad \text { for } i \in\{1,2\}
$$

and one obtains from 2.1 for $\mathscr{L}^{m}$ almost all $x \in \mathbf{U}(0,1)$

$$
\begin{gathered}
\left\langle\mathbf{D}^{2} u_{i}(x), C_{F}\left(\mathbf{D} u_{i}(x)\right)\right\rangle=0 \quad \text { for } i \in\{1,2\}, \\
\left\langle\mathbf{D}^{2}\left(u_{2}-u_{1}\right)(x), C_{F}\left(\mathbf{D} u_{2}(x)\right)\right\rangle=\left\langle\mathbf{D}^{2} u_{1}(x), C_{F}\left(\mathbf{D} u_{1}(x)\right)-C_{F}\left(\mathbf{D} u_{2}(x)\right)\right\rangle .
\end{gathered}
$$

Therefore by 2.4, 2.1 and Hölder's inequality

$$
\begin{aligned}
\left|\mathbf{D}^{2}\left(u_{2}-u_{1}\right)\right|_{a, 1 / 4 ; p} \leq \Delta_{2}\left(2^{2+m-m / p}\right. & \left|u_{2}-u_{1}\right|_{0,1 / 2 ; 1} \\
& \left.+\kappa \Lambda\left|\mathbf{D}^{2} u_{1}\right|_{2 p ; 0,1 / 2}\left|\mathbf{D}\left(u_{2}-u_{1}\right)\right|_{2 p ; 0,1 / 2}\right) .
\end{aligned}
$$

To estimate $\left|\mathbf{D}\left(u_{2}-u_{1}\right)\right|_{2 p ; 0,1 / 2}$, one computes for $\theta \in \mathscr{D}\left(\mathbf{U}(0,1), \mathbf{R}^{n-m}\right)$

$$
\begin{gathered}
-\int_{\mathbf{U}(0,1)}\left\langle D \theta(x) \odot \mathbf{D}\left(u_{2}-u_{1}\right)(x), A(x)\right\rangle \mathrm{d} \mathscr{L}^{m} x=0, \\
\text { where } A(x)=\int_{0}^{1} D^{2} F\left(t \mathbf{D} u_{2}(x)+(1-t) \mathbf{D} u_{1}(x)\right) \mathrm{d} \mathscr{L}^{1} t,
\end{gathered}
$$

and obtains from 2.3

$$
\left|\mathbf{D}\left(u_{2}-u_{1}\right)\right|_{2 p ; 0,1 / 2} \leq \Delta_{3}\left|u_{2}-u_{1}\right|_{1 ; 0,1}
$$

and the conclusion follows.

2.8 Lemma. Suppose $m, n \in \mathscr{P}, m<n, 1 \leq p \leq r<\infty$, and $1<q<\infty$.

Then there exist a positive, finite number $\varepsilon$, a positive, finite number $\Gamma_{1}$ depending only on $m$ and $p$, and a positive, finite number $\Gamma_{2}$ depending only on $m, n, p$, and $r$ with the following property.

If $F$ is related to $\varepsilon$ as in 2.1. Lip $D^{2} F<\infty, j \in\{0,1\}, A$ is a closed subset of $\mathbf{R}^{m}, u: \mathbf{R}^{m} \cap\{x: \operatorname{dist}(x, A)<1\} \rightarrow \mathbf{R}^{n-m}$ is $j$ times weakly differentiable, $0 \leq$ $\gamma<\infty$, and if for each $a \in A, 0<\varrho \leq 1$ there are $v_{a, \varrho} \in \mathbf{W}^{1, q}\left(\mathbf{U}(a, \varrho), \mathbf{R}^{n-m}\right)$ and an affine function $P_{a, \varrho}: \mathbf{R}^{m} \rightarrow \mathbf{R}^{n-m}$ such that

$$
\begin{gathered}
L_{F}\left(v_{a, \varrho}\right)=0, \\
\sum_{i=0}^{j} \varrho^{-m / p+i}\left|\mathbf{D}^{i}\left(u-v_{a, \varrho}\right)\right|_{p ; a, \varrho} \leq \gamma \varrho^{2}, \quad \varrho^{-m / p}\left|u-P_{a, \varrho}\right|_{p ; a, \varrho} \leq \gamma \varrho
\end{gathered}
$$

then there exists a twice weakly differentiable function $v: \mathbf{R}^{m} \cap\{x: \operatorname{dist}(x, A)<$ $\left.\frac{1}{36}\right\} \rightarrow \mathbf{R}^{n-m}$ with

$$
\begin{gathered}
\sum_{i=0}^{j} \varrho^{-m / p+i}\left|\mathbf{D}^{i}(u-v)\right|_{p ; a, \varrho} \leq \Gamma_{1} \gamma \varrho^{2}, \\
\varrho^{-m / r}\left|\mathbf{D}^{2} v\right|_{r ; a, \varrho} \leq \Gamma_{2}\left(\gamma\left(1+\operatorname{Lip}\left(D^{2} F\right) \gamma\right)^{2}+\varrho^{-m-2}\left|u-P_{a, 2 \varrho}\right|_{1 ; a, 2 \varrho}\right)
\end{gathered}
$$

whenever $a \in A, 0<\varrho \leq \frac{1}{36}$. 
Proof. Assume $r \geq q$ and define

$$
\varepsilon=\inf \{1, q 2.5(n, q, 2 r), q 2.7(n, q, 2 r), 92.5(n, q, r)\} .
$$

Suppose $F, j, A, u, \gamma, v_{a, \varrho}$, and $P_{a, \varrho}$ are as in the hypotheses in the body of the lemma with $\varepsilon$ and abbreviate $\Lambda=\operatorname{Lip} D^{2} F$.

By 2.5 and Hölder's inequality

$$
\sum_{i=0}^{j}\left|\mathbf{D}^{i} v_{a, \varrho}\right|_{2 r ; a, 1 / 2}<\infty, \quad \sum_{i=0}^{j}\left|\mathbf{D}^{i} u\right|_{p ; a, 1 / 2}<\infty
$$

whenever $a \in A$. Therefore taking limits (for example by use of an interpolation inequality similar to Mor66, Lemma 6.2.2] and weak compactness properties of Sobolev spaces [Mor66, Theorem 3.2.4(e)]) the conclusion can be deduced from the following assertion: There exist a positive, finite number $\Gamma_{1}$ depending only on $m$ and $p$, and a positive, finite number $\Gamma_{2}$ depending only on $m, n, p$ and $r$ such that for every $0<\delta \leq \frac{1}{18}$ there exists a function $v: \mathbf{R}^{m} \rightarrow \mathbf{R}^{n-m}$ whose restriction to $\mathbf{R}^{m} \cap\left\{x: \operatorname{dist}(x, A)<\frac{1}{18}\right\}$ is twice weakly differentiable satisfying

$$
\begin{gathered}
\sum_{i=0}^{j} \varrho^{-m / p+i}\left|\mathbf{D}^{i}(u-v)\right|_{p ; a, \varrho} \leq \Gamma_{1} \gamma \varrho^{2} \\
(\varrho / 2)^{-m / r}\left|\mathbf{D}^{2} v\right|_{r ; a, \varrho / 2} \leq \Gamma_{2}\left(\gamma(1+\Lambda \gamma)^{2}+(\varrho / 2)^{-m-2}\left|u-P_{a, \varrho}\right|_{1 ; a, \varrho}\right)
\end{gathered}
$$

whenever $a \in A, \delta \leq \varrho \leq \frac{1}{18}$.

Assume $A \neq \emptyset$, let $\Phi \stackrel{=}{=}\left\{\mathbf{R}^{m} \sim A\right\} \cup\{\mathbf{U}(a, \delta): a \in A\}$, note $\bigcup \Phi=\mathbf{R}^{m}$, define $h: \mathbf{R}^{m} \rightarrow \mathbf{R}$ by

$$
h(x)=\frac{1}{20} \sup \left\{\inf \left\{1, \operatorname{dist}\left(x, \mathbf{R}^{m} \sim U\right)\right\}: U \in \Phi\right\} \quad \text { for } x \in \mathbf{R}^{m},
$$

and apply Fed69, 3.1.13] to obtain a countable subset $S$ of $\mathbf{R}^{m}$ and functions $\varphi_{s}: \mathbf{R}^{m} \rightarrow\{t: 0 \leq t \leq 1\}$ of class $\infty$ corresponding to $s \in S$ such that with $S_{x}=S \cap\{s: \mathbf{B}(x, 10 h(x)) \cap \mathbf{B}(s, 10 h(s)) \neq \emptyset\}$ for $x \in \mathbf{R}^{m}$ and a sequence $V_{i}$ of positive, finite numbers depending only on $m$ there holds

$$
\operatorname{card} S_{x} \leq(129)^{m}, \quad \operatorname{spt} \varphi_{s} \subset \mathbf{B}(s, 10 h(s)) \text { for } s \in S,
$$

$1 / 3 \leq h(x) / h(s) \leq 3 \quad$ for $s \in S_{x}, \quad\left|D^{i} \varphi_{s}(x)\right| \leq V_{i}(h(x))^{-i} \quad$ for $s \in S, i \in \mathscr{P}$,

$$
\sum_{s \in S} \varphi_{s}(y)=\sum_{s \in S_{x}} \varphi_{s}(y)=1, \quad \sum_{s \in S} D^{i} \varphi_{s}(y)=\sum_{s \in S_{x}} D^{i} \varphi_{s}(y)=0 \quad \text { for } i \in \mathscr{P}
$$

whenever $x \in \mathbf{R}^{m}, y \in \mathbf{B}(x, 10 h(x))$. Note for $x \in \mathbf{R}^{m}, y \in \mathbf{B}(x, 10 h(x)), s \in S$, $i \in \mathscr{P}$

$$
\left|D^{i} \varphi_{s}(y)\right| \leq V_{i}(h(y))^{-i} \leq(20)^{i} V_{i}(10 h(x))^{-i},
$$

because $h(x)-h(y) \leq \frac{1}{20}|x-y| \leq \frac{1}{2} h(x)$. Choose $\xi: S \rightarrow A$ such that

$$
|\xi(s)-s|=\operatorname{dist}(s, A) \quad \text { whenever } s \in S .
$$

Note $20 h(x) \leq \sup \{\operatorname{dist}(x, A), \delta\}$ for $x \in \mathbf{R}^{m}$ and observe

$$
\mathbf{B}(x, 20 h(x)) \subset \mathbf{B}(\xi(s), 120 h(s)), \quad 120 h(s) \leq 1
$$


whenever $x \in \mathbf{R}^{m}$, $\operatorname{dist}(x, A) \leq \frac{1}{18}, s \in S_{x}$, because

$$
\begin{gathered}
|x-s| \leq 10 h(x)+10 h(s) \leq 40 h(x) \leq 2 \sup \{\operatorname{dist}(x, A), \delta\} \leq 1 / 9, \\
|s-\xi(s)|=\operatorname{dist}(s, A) \leq|x-s|+\operatorname{dist}(x, A) \leq 1 / 6, \\
|x-\xi(s)| \leq|x-s|+|s-\xi(s)| \leq 40 h(s)+20 h(s)=60 h(s), \\
|x-\xi(s)|+20 h(x) \leq 120 h(s) \leq 360 h(x) \leq 1 .
\end{gathered}
$$

Define $R=\bigcup\left\{S_{x}: x \in \mathbf{R}^{m}\right.$ and $\left.\operatorname{dist}(x, A) \leq \frac{1}{18}\right\}$,

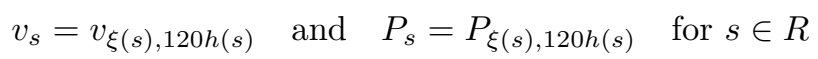

and, denoting by $v_{s}^{\prime}$ the extension of $v_{s}$ to $\mathbf{R}^{m}$ by $0, v: \mathbf{R}^{m} \rightarrow \mathbf{R}^{n-m}$ by

$$
v(x)=\sum_{s \in R} \varphi_{s}(x) v_{s}^{\prime}(x) \quad \text { whenever } x \in \mathbf{R}^{m} .
$$

Suppose for the rest of the proof $x \in \mathbf{R}^{m}$ with $\operatorname{dist}(x, A) \leq \frac{1}{18}$ and observe

$$
v(y)=\sum_{s \in S_{x}} \varphi_{s}(y) v_{s}(y) \quad \text { whenever } y \in \mathbf{B}(x, 10 h(x)) .
$$

The asserted weak differentiability is a consequence of 2.5

One estimates

$$
\begin{aligned}
& \left|\mathbf{D}^{i}\left(u-v_{s}\right)\right|_{p ; x, 20 h(x)} \leq\left|\mathbf{D}^{i}\left(u-v_{s}\right)\right|_{p ; s, 120 h(s)} \\
& \quad \leq \gamma(120 h(s))^{m / p+2-i} \leq(18)^{m / p+2} \gamma(20 h(x))^{m / p+2-i}
\end{aligned}
$$

for $i \in\{0, j\}, s \in S_{x}$, hence by Hölder's inequality

$$
\begin{aligned}
& (20 h(x))^{-m}\left|u-v_{s}\right|_{1 ; x, 20 h(x)} \\
\leq & \boldsymbol{\alpha}(m)^{1-1 / p} \sum_{i=0}^{j}(20 h(x))^{-m / p+i}\left|\mathbf{D}^{i}\left(u-v_{s}\right)\right|_{p ; x, 20 h(x)} \leq 2 \Delta_{1} \gamma(20 h(x))^{2}
\end{aligned}
$$

for $s \in S_{x}$ where $\Delta_{1}=\boldsymbol{\alpha}(m)^{1-1 / p}(18)^{m / p+2}$. Also

$$
\begin{aligned}
&(20 h(x))^{-m}\left|u-P_{s}\right|_{1 ; x, 20 h(x)} \leq \boldsymbol{\alpha}(m)^{1-1 / p}(20 h(x))^{-m / p}\left|u-P_{s}\right|_{p ; \xi(s), 120 h(s)} \\
& \leq \Delta_{1} \gamma(20 h(x)), \\
&(20 h(x))^{-m}\left|v_{s}-P_{s}\right|_{1 ; x, 20 h(x)} \leq 3 \Delta_{1} \gamma(20 h(x))
\end{aligned}
$$

for $s \in S_{x}$. Using

$$
v(y)-u(y)=\sum_{s \in S_{x}} \varphi_{s}(y)\left(v_{s}(y)-u(y)\right) \quad \text { whenever } y \in \mathbf{B}(x, 10 h(x))
$$

and the Leibnitz formula, one obtains from (I)

$$
\sum_{i=0}^{j}(10 h(x))^{-m / p+i}\left|\mathbf{D}^{i}(u-v)\right|_{p ; x, 10 h(x)} \leq \Delta_{2} \gamma(10 h(x))^{2}
$$

where $\Delta_{2}=\boldsymbol{\alpha}(m)^{1 / p-1} 8 \Delta_{1} 2^{m / p}\left(1+20 V_{1}\right)(129)^{m}$.

In case $x \in \mathbf{B}(a, \varrho)$ for some $a \in A, \delta \leq \varrho \leq \frac{1}{18}$,

$$
20 h(x) \leq \sup \{\operatorname{dist}(x, A), \delta\} \leq \varrho, \quad \mathbf{B}(x, 20 h(x)) \subset \mathbf{B}(a, 2 \varrho)
$$


and Vitali's covering theorem yields a countable subset $T$ of $\mathbf{B}(a, \varrho)$ such that

$$
\{\mathbf{B}(t, 2 h(t)): t \in T\} \text { is disjointed, } \mathbf{B}(a, \varrho) \subset \bigcup\{\mathbf{B}(t, 10 h(t)): t \in T\}
$$

and one estimates for $i \in\{0, j\}$

$$
\begin{aligned}
\mid \mathbf{D}^{i}(u & -v)\left.\right|_{p ; a, \varrho} ^{p} \\
& \leq \sum_{t \in T}\left|\mathbf{D}^{i}(u-v)\right|_{p ; t, 10 h(t)}^{p} \\
& \leq\left(\Delta_{2} \gamma\right)^{p} \sum_{t \in T}(10 h(t))^{m+(2-i) p} \\
& =\left(5^{m / p+2-i} \Delta_{2} \gamma\right)^{p} \boldsymbol{\alpha}(m)^{-1-(2-i) p / m} \sum_{t \in T} \mathscr{L}^{m}(\mathbf{B}(t, 2 h(t)))^{1+(2-i) p / m} \\
& \leq\left(5^{m / p+2-i} \Delta_{2} \gamma\right)^{p} \boldsymbol{\alpha}(m)^{-1-(2-i) p / m} \mathscr{L}^{m}(\mathbf{B}(a, 2 \varrho))^{1+(2-i) p / m} \\
& =\left((10)^{m / p+2-i} \Delta_{2} \gamma\right)^{p} \varrho^{m+(2-i) p} .
\end{aligned}
$$

Therefore one obtains for $a \in A, \delta \leq \varrho \leq \frac{1}{18}, i \in\{0, j\}$

$$
\varrho^{-m / p+i}\left|\mathbf{D}^{i}(u-v)\right|_{p ; a, \varrho} \leq(10)^{m / p+2} \Delta_{2} \gamma \varrho^{2}
$$

and one may take $\Gamma_{1}=2(10)^{m / p+2} \Delta_{2}$ in the first estimate of the assertion.

According to 2.5 the functions $v_{s}$ are twice weakly differentiable and satisfy for $s \in S_{x}$

$$
(20 h(x))^{-m /(2 r)+2}\left|\mathbf{D}^{2} v_{s}\right|_{2 r ; x, 10 h(x)} \leq \Delta_{3}(20 h(x))^{-m}\left|v_{s}-P_{s}\right|_{1 ; x, 20 h(x)}
$$

where $\Delta_{3}=\Gamma[2.5](n, 2 r)$. Combining this with (III) yields

$$
(10 h(x))^{-m /(2 r)+2}\left|\mathbf{D}^{2} v_{s}\right|_{2 r ; x, 10 h(x)} \leq 2^{m /(2 r)} 3 \Delta_{1} \Delta_{3} \gamma(10 h(x))
$$

for $s \in S_{x}$.

Using 2.7 one obtains for $s, t \in S_{x}$

$$
\begin{gathered}
(20 h(x))^{-m /(2 r)+1}\left|\mathbf{D}^{2}\left(v_{s}-v_{t}\right)\right|_{2 r ; x, 10 h(x)} \leq \Delta_{4}\left((20 h(x))^{-m-1}\left|v_{s}-v_{t}\right|_{1 ; x, 20 h(x)}\right. \\
\left.\quad+\Lambda\left((20 h(x))^{-m-1}\left|v_{s}-P_{s}\right|_{1 ; x, 20 h(x)}\right)\left((20 h(x))^{-m-1}\left|v_{s}-v_{t}\right|_{1 ; x, 20 h(x)}\right)\right)
\end{gathered}
$$

where $\Delta_{4}=\Gamma_{2.7}(n, 2 r)$. Since

$$
(20 h(x))^{-m}\left|v_{s}-v_{t}\right|_{1 ; x, 20 h(x)} \leq 4 \Delta_{1} \gamma(20 h(x))^{2}
$$

by (I) , one estimates using (II)

$$
(10 h(x))^{-m /(2 r)}\left|\mathbf{D}^{2}\left(v_{s}-v_{t}\right)\right|_{2 r ; x, 10 h(x)} \leq \Delta_{5} \gamma(1+\Lambda \gamma)
$$

where $\Delta_{5}=2^{m+2} \Delta_{1} \Delta_{4} \sup \left\{3 \Delta_{1}, 1\right\}$. Using an interpolation inequality (which may be proven similarly to [Mor66, Lemma 6.2.2]), one infers with a positive, finite number $\Delta_{6}$ depending only $n$ and $r$

$$
\begin{aligned}
\sum_{i=0}^{2} & (10 h(x))^{-m /(2 r)+i}\left|\mathbf{D}^{i}\left(v_{s}-v_{t}\right)\right|_{2 r ; x, 10 h(x)} \\
\leq & \Delta_{6}\left((10 h(x))^{-m /(2 r)+2}\left|\mathbf{D}^{2}\left(v_{s}-v_{t}\right)\right|_{2 r ; x, 10 h(x)}\right. \\
& \left.+(10 h(x))^{-m}\left|v_{s}-v_{t}\right|_{1 ; x, 10 h(x)}\right) \\
\leq & \Delta_{6}\left(\Delta_{5}(1+\Lambda \gamma)+2^{m+4} \Delta_{1}\right) \gamma(10 h(x))^{2} .
\end{aligned}
$$


This implies for $s, t \in S_{x}$

$$
\sum_{i=0}^{2}(10 h(x))^{-m /(2 r)+i}\left|\mathbf{D}^{i}\left(v_{s}-v_{t}\right)\right|_{2 r ; x, 10 h(x)} \leq \Delta_{7} \gamma(1+\Lambda \gamma)(10 h(x))^{2}
$$

where $\Delta_{7}=\Delta_{6}\left(\Delta_{5}+2^{m+4} \Delta_{1}\right)$. Noting $\left(v-v_{s}\right)(y)=\sum_{t \in S_{x}} \varphi_{t}(y)\left(v_{t}-v_{s}\right)(y)$ for $s \in S_{x}, y \in \mathbf{U}(x, 10 h(x))$, one infers using the Leibnitz formula

$$
(10 h(x))^{-m /(2 r)+i}\left|\mathbf{D}^{i}\left(v-v_{s}\right)\right|_{2 r ; x, 10 h(x)} \leq \Delta_{8} \gamma(1+\Lambda \gamma)(10 h(x))^{2}
$$

for $s \in S_{x}, i \in\{0,1,2\}$ where $\Delta_{8}=2\left(1+20 V_{1}+400 V_{2}\right) \Delta_{7}(129)^{m}$.

Using 2.1, one defines

$$
f(y)=\left\langle\mathbf{D}^{2} v(y), C_{F}(\mathbf{D} v(y))\right\rangle
$$

whenever $y \in \mathbf{U}(z, 10 h(z))$ for some $z \in \mathbf{R}^{m}$ with $\operatorname{dist}(z, A) \leq \frac{1}{18}$ and computes for $s \in S_{x}$

$$
f(y)=\left\langle\mathbf{D}^{2} v_{s}(y), C_{F}(\mathbf{D} v(y))-C_{F}\left(\mathbf{D} v_{s}(y)\right)\right\rangle+\left\langle\mathbf{D}^{2}\left(v-v_{s}\right)(y), C_{F}(\mathbf{D} v(y))\right\rangle
$$

for $\mathscr{L}^{m}$ almost all $y \in \mathbf{U}(x, 10 h(x))$. Hölder's inequality implies

$$
\begin{aligned}
|f|_{r ; x, 10 h(x)} \leq & \kappa \Lambda\left|\mathbf{D}\left(v-v_{s}\right)\right|_{2 r ; x, 10 h(x)}\left|\mathbf{D}^{2} v_{s}\right|_{2 r ; x, 10 h(x)} \\
& +2 \kappa \boldsymbol{\alpha}(m)^{1 /(2 r)}(10 h(x))^{m /(2 r)}\left|\mathbf{D}^{2}\left(v-v_{s}\right)\right|_{2 r ; x, 10 h(x)},
\end{aligned}
$$

hence by (IV) and (V)

$$
(10 h(x))^{-m / r}|f|_{r ; x, 10 h(x)} \leq \Delta_{9} \gamma(1+\Lambda \gamma)^{2}
$$

where $\Delta_{9}=\kappa \Delta_{8} \sup \left\{2^{m /(2 r)} 3 \Delta_{1} \Delta_{3}, 2 \boldsymbol{\alpha}(m)^{1 /(2 r)}\right\}$. Similarly but simpler as in the deduction of (III), one obtains for $\delta \leq \varrho \leq \frac{1}{18}, a \in A$

$$
|f|_{r ; a, \varrho} \leq \Delta_{9}(10)^{m / r} \gamma(1+\Lambda \gamma)^{2} \varrho^{m / r}
$$

and thus, using 2.5 with $\Delta_{10}=\Gamma_{2.5}(n, r)$ and (III),

$$
\begin{aligned}
\varrho^{-m / r}\left|\mathbf{D}^{2} v\right|_{r ; a, \varrho / 2} & \leq \Delta_{10}\left(\varrho^{-m-2}\left(|u-v|_{1 ; a, \varrho}+\left|u-P_{a, \varrho}\right|_{1 ; a, \varrho}\right)+\varrho^{-m / r}|f|_{r ; a, \varrho}\right) \\
& \leq \Delta_{11}\left(\gamma(1+\Lambda \gamma)^{2}+\varrho^{-m-2}\left|u-P_{a, \varrho}\right|_{1 ; a, \varrho}\right)
\end{aligned}
$$

where $\Delta_{11}=\Delta_{10}\left(\boldsymbol{\alpha}(m)^{1-1 / p}(10)^{m / p+2} \Delta_{2}+\Delta_{9}(10)^{m / r}+1\right)$. Therefore one may take $\Gamma_{2}=2^{m / r} \Delta_{11}$ in the second estimate of the assertion and the proof is completed.

2.9 Remark. In fact, by Calderón and Zygmund [CZ61, Theorem 10 (ii)] (see also [Zie89, Lemma 3.7.2]) or by [Men09a, 3.1]

$$
\lim _{\varrho \rightarrow 0+} \varrho^{-2} \sum_{i=0}^{j} \varrho^{-m / p+i}\left|\mathbf{D}^{i}(u-v)\right|_{p ; a, \varrho}=0
$$

for $\mathscr{L}^{m}$ almost all $a \in A$. Now, Rešetnyak's result in Reš68 applied to $v$ yields that for $\mathscr{L}^{m}$ almost all $a \in A$ there exists a polynomial function $Q_{a}: \mathbf{R}^{m} \rightarrow$ $\mathbf{R}^{n-m}$ of degree at most 2 such that

$$
\limsup _{\varrho \rightarrow 0+} \varrho^{-2} \sum_{i=0}^{j} \varrho^{-m / p+i}\left|\mathbf{D}^{i}\left(u-Q_{a}\right)\right|_{p ; a, \varrho}=0 .
$$

Alternately, this latter fact could have also been deduced by use of Calderón and Zygmund [CZ61, Theorem 12] (see also Zie89, Theorem 3.4.2]). 
2.10 Theorem. Suppose $m, n \in \mathscr{P}, m<n, 1 \leq p<\infty$, and $1<q<\infty$.

Then there exists a positive, finite number $\varepsilon$ with the following property.

If $F$ is related to $\varepsilon$ as in 2.1, Lip $D^{2} F<\infty, U$ is an open subset of $\mathbf{R}^{m}$, $j \in\{0,1\}, u: U \rightarrow \mathbf{R}^{n-m}$ is weakly differentiable,

$$
\begin{gathered}
h(a, r)= \\
\inf \left\{\sum_{i=0}^{j} r^{-m / p+i}\left|\mathbf{D}^{i}(u-v)\right|_{p ; a, r}: v \in \mathbf{W}^{1, q}\left(\mathbf{U}(a, r), \mathbf{R}^{n-m}\right) \text { and } L_{F}(v)=0\right\}
\end{gathered}
$$

whenever $\mathbf{U}(a, r) \subset U$ for some $a \in \mathbf{R}^{m}, 0<r<\infty$, and if $A$ denotes the set of all $a \in U$ such that

$$
\limsup _{r \rightarrow 0+} r^{-2} h(a, r)<\infty,
$$

then $A$ is a Borel set and for $\mathscr{L}^{m}$ almost all a $\in A$ there exists a polynomial function $Q_{a}: \mathbf{R}^{m} \rightarrow \mathbf{R}^{n-m}$ with degree at most 2 such that

$$
\lim _{r \rightarrow 0+} r^{-2} \sum_{i=0}^{j} r^{-m / p+i}\left|\mathbf{D}^{i}\left(u-Q_{a}\right)\right|_{p ; a, r}=0 .
$$

Proof. In view of 2.5 one may assume $q \geq p$. Let $\varepsilon=q 2.8(m, n, p, p, q)$. Suppose $F, U, j$, and $u$ satisfy the hypotheses with $\varepsilon$. Define the open set $V$ by

$$
V=U \cap\left\{x: \sum_{i=0}^{j}\left|\mathbf{D}^{i} u\right|_{p ; x, r}<\infty \text { for some } 0<r<\operatorname{dist}\left(x, \mathbf{R}^{m} \sim U\right)\right\}
$$

and note $A \subset V$. Denote by $D$ the set of all $v \in \mathbf{W}^{1, q}\left(\mathbf{U}(0,1), \mathbf{R}^{n-m}\right)$ such that $L_{F}(v)=0$ and define

$$
W=(V \times \mathbf{R}) \cap\left\{(a, r): 0<r<\operatorname{dist}\left(a, \mathbf{R}^{m} \sim V\right)\right\}
$$

and the continuous map $T: W \rightarrow \mathbf{W}^{1, p}\left(\mathbf{U}(0,1), \mathbf{R}^{n-m}\right)$ by

$$
T(a, r)(x)=r^{-1} u(a+r x) \quad \text { whenever }(a, r) \in W, x \in \mathbf{U}(0,1) .
$$

Since $D \neq \emptyset$ and

$$
h(a, r)=r \inf \left\{\sum_{i=0}^{j}\left|\mathbf{D}^{i}(T(a, r)-v)\right|_{p ; 0,1}: v \in D\right\} \quad \text { for }(a, r) \in W,
$$

$h$ is continuous. Therefore $A$ is a Borel set. Similarly, denoting by $D^{\prime}$ the set of all affine functions mapping $\mathbf{R}^{m}$ into $\mathbf{R}^{n-m}$ one defines a continuous map $h^{\prime}: W \rightarrow \mathbf{R}$ by

$$
h^{\prime}(a, r)=r \inf \left\{|T(a, r)-w|_{1 ; 0,1}: w \in D^{\prime}\right\} \quad \text { for }(a, r) \in W .
$$

By Rešetnyak [Reš68] or [Fed69, 4.5.9 (26)(II) (III)] one notes

$$
\underset{\varrho \rightarrow 0+}{\limsup } \varrho^{-1} h^{\prime}(a, \varrho)<\infty \quad \text { for } \mathscr{L}^{m} \text { almost all } a \in U \text {. }
$$

Define

$$
\begin{gathered}
C_{k}=V \cap\left\{x: \operatorname{dist}\left(x, \mathbf{R}^{m} \sim V\right) \geq 1 / k\right\}, \\
A_{k}=C_{k} \cap\left\{a: h(a, r) \leq k r^{2} \text { and } h^{\prime}(a, r) \leq k r \text { for } 0<r<1 / k\right\}
\end{gathered}
$$

for $k \in \mathscr{P}$ and observe that the sets $A_{k}$ are closed and

$$
\mathscr{L}^{m}\left(A \sim \bigcup\left\{A_{k}: k \in \mathscr{P}\right\}\right)=0 .
$$

Finally, the conclusion is obtained by applying (for each $k \in \mathscr{P}$ ) 2.8 in conjunction with 2.9 to rescaled versions of $u, A_{k}$ and a suitable number $\gamma$. 
2.11 Remark. Instead of using Rešetnyak Reš68] or [Fed69, 4.5.9 (26) (II) (III)], one can also use the functions $v$ occurring in the definition of $h(a, r)$ in a way reminiscent of the familiar harmonic approximation procedure to deduce

$$
\limsup _{\varrho \rightarrow 0+} \varrho^{-1} h^{\prime}(a, \varrho)<\infty \quad \text { whenever } a \in A .
$$

Therefore $u$ could have been required to be merely $j$ times weakly differentiable.

2.12 Corollary. Suppose $m, n \in \mathscr{P}, m<n, 1<p<\infty, U$ is an open subset of $\mathbf{R}^{m}, T \in \mathscr{D}\left(U, \mathbf{R}^{n-m}\right)$ and $A$ denotes the set of all $a \in U$ such that

$$
\limsup _{r \rightarrow 0+} r^{-1-m / p}|T|_{-1, p ; a, r}<\infty .
$$

Then $A$ is a Borel set and for $\mathscr{L}^{m}$ almost all $a \in A$ there exists a unique constant distribution $T_{a} \in \mathscr{D}^{\prime}\left(U, \mathbf{R}^{n-m}\right)$ such that

$$
\lim _{r \rightarrow 0+} r^{-1-m / p}\left|T-T_{a}\right|_{-1, p ; a, r}=0 .
$$

Proof. The conclusion is local and for each $a \in A$ there exists $0<r<\infty$ with $|T|_{-1, p ; a, r}<\infty$, hence one may assume spt $T$ to be compact, $U=\mathbf{R}^{m}$ and $|T|_{-1, p ; 0, R}<\infty$, spt $T \subset \mathbf{U}(0, R)$ for some $0<R<\infty$.

For example using 2.2, one obtains functions $u \in \mathbf{W}_{0}^{1, p}\left(\mathbf{U}(0, R), \mathbf{R}^{n-m}\right)$ and $v_{a, r} \in \mathscr{E}\left(\mathbf{U}(a, r), \mathbf{R}^{n-m}\right)$ whenever $a \in \mathbf{R}^{m}, 0<r<\infty$ and $\mathbf{U}(a, r) \subset \mathbf{U}(0, R)$ such that

$$
\begin{gathered}
-\int_{\mathbf{U}(0, R)} \mathbf{D} u \bullet D \theta \mathrm{d} \mathscr{L}^{m}=T(\theta) \quad \text { for } \theta \in \mathscr{D}\left(\mathbf{U}(0, R), \mathbf{R}^{n-m}\right), \\
u-v_{a, r} \in \mathbf{W}_{0}^{1, p}\left(\mathbf{U}(a, r), \mathbf{R}^{n-m}\right), \quad \operatorname{Lap} v_{a, r}=0 .
\end{gathered}
$$

By 2.2 and Poincaré's inequality

$$
\sum_{i=0}^{1} r^{i-1}\left|\mathbf{D}^{i}\left(u-v_{a, r}\right)\right|_{p ; a, r} \leq \Delta|T|_{-1, p ; a, r}
$$

for some positive, finite number $\Delta$ depending only on $n$ and $p$, hence the set $A$ agrees with the set " $A$ " defined in 2.10 with $q=p, F$ the Dirichlet integrand and $j=1$. Therefore, applying 2.10 , one may take $T_{a} \in \mathscr{D}^{\prime}\left(\mathbf{U}(0, R), \mathbf{R}^{n-m}\right)$ defined by $T_{a}(\theta)=\int \theta(x) \bullet \operatorname{Lap} Q_{a}(a) \mathrm{d} \mathscr{L}^{m} x$ for $\theta \in \mathscr{D}\left(\mathbf{R}^{m}, \mathbf{R}^{n-m}\right)$.

The uniqueness follows, since every $T_{a}$ admissible in the conclusion satisfies $r^{-m} T_{a}\left(\theta \circ \boldsymbol{\mu}_{1 / r} \circ \boldsymbol{\tau}_{-a}\right)=T_{a}(\theta), \quad r^{-m} T\left(\theta \circ \boldsymbol{\mu}_{1 / r} \circ \boldsymbol{\tau}_{-a}\right) \rightarrow T_{a}(\theta) \quad$ as $r \rightarrow 0+$. whenever $\theta \in \mathscr{D}\left(\mathbf{R}^{m}, \mathbf{R}^{n-m}\right)$.

2.13 Lemma. Suppose $m, n \in \mathscr{P}, m<n, \Phi \in \bigodot^{2} \operatorname{Hom}\left(\mathbf{R}^{m}, \mathbf{R}^{n-m}\right), 0<$ $c \leq M<\infty,\|\Phi\| \leq M, \Phi$ is strongly elliptic with ellipticity bound $c, a \in \mathbf{R}^{m}$, $0<r<\infty, u \in \mathbf{W}_{0}^{1,1}\left(\mathbf{U}(a, r), \mathbf{R}^{n-m}\right), T \in \mathscr{D}^{\prime}\left(\mathbf{U}(a, r), \mathbf{R}^{n-m}\right)$, and

$$
-\int_{\mathbf{U}(a, r)}\langle D \theta(x) \odot \mathbf{D} u(x), \Phi\rangle \mathrm{d} \mathscr{L}^{m} x=T(\theta) \quad \text { for } \theta \in \mathscr{D}\left(\mathbf{U}(a, r), \mathbf{R}^{n-m}\right) .
$$

Then

$$
|u|_{1 ; a, r} \leq \operatorname{\Gamma r}|T|_{-1,1 ; a, r}
$$

where $\Gamma$ is a positive, finite number depending only on $n, c$, and $M$. 
Proof. See Men09b, 6.8].

2.14 Lemma. Suppose $m, n \in \mathscr{P}, m<n, 0<c \leq M<\infty$,

$$
F: \operatorname{Hom}\left(\mathbf{R}^{m}, \mathbf{R}^{n-m}\right) \rightarrow \mathbf{R} \text { is of class } 2,
$$

$$
\left\|D^{2} F(\sigma)\right\| \leq M, \quad\left\langle(\tau, \tau), D^{2} F(\sigma)\right\rangle \geq c|\tau|^{2} \quad \text { for } \sigma, \tau \in \operatorname{Hom}\left(\mathbf{R}^{m}, \mathbf{R}^{n-m}\right),
$$

$a \in \mathbf{R}^{m}, 0<r<\infty$, and $u, v \in \mathbf{W}^{1,2}\left(\mathbf{U}(a, r), \mathbf{R}^{n-m}\right)$ with

$$
u-v \in \mathbf{W}_{0}^{1,2}\left(\mathbf{U}(a, r), \mathbf{R}^{n-m}\right) .
$$

Then for every affine function $P: \mathbf{R}^{m} \rightarrow \mathbf{R}^{n-m}$

$$
|\mathbf{D}(v-u)|_{2 ; a, r} \leq c^{-1}\left(M|\mathbf{D}(u-P)|_{2 ; a, r}+\left|L_{F}(v)\right|_{-1,2 ; a, r}\right)
$$

where $L_{F}$ is defined as in 2.1.

Proof. Compute for $\theta \in \mathscr{D}\left(\mathbf{U}(a, r), \mathbf{R}^{n-m}\right)$

$$
\begin{gathered}
L_{F}(v)(\theta)=-\int_{\mathbf{U}(a, r)}\langle D \theta(x), D F(\mathbf{D} v(x))-D F(D P(x))\rangle \mathrm{d} \mathscr{L}^{m} x \\
=-\int_{\mathbf{U}(a, r)}\langle D \theta(x) \odot \mathbf{D}(v-P)(x), A(x)\rangle \mathrm{d} \mathscr{L}^{m} x \\
\text { where } A(x)=\int_{0}^{1} D^{2} F(t \mathbf{D} v(x)+(1-t) D P(x)) \mathrm{d} \mathscr{L}^{1} t .
\end{gathered}
$$

This implies for $\theta \in \mathscr{D}\left(\mathbf{U}(a, r), \mathbf{R}^{n-m}\right)$

$$
\begin{aligned}
& \int_{\mathbf{U}(a, r)}\langle D \theta(x) \odot \mathbf{D}(v-u)(x), A(x)\rangle \mathrm{d} \mathscr{L}^{m} x \\
& \quad=-\int_{\mathbf{U}(a, r)}\langle D \theta(x) \odot \mathbf{D}(u-P)(x), A(x)\rangle \mathrm{d} \mathscr{L}^{m} x-L_{F}(v)(\theta) .
\end{aligned}
$$

Letting $\theta$ approximate $v-u$ in $\mathbf{W}^{1,2}\left(\mathbf{U}(a, r), \mathbf{R}^{n-m}\right)$, one obtains

$$
c\left(|\mathbf{D}(v-u)|_{2 ; a, r}\right)^{2} \leq\left(M|\mathbf{D}(u-P)|_{2 ; a, r}+\left|L_{F}(v)\right|_{-1,2 ; a, r}\right)|\mathbf{D}(v-u)|_{2 ; a, r} .
$$

2.15 Lemma. Suppose $m, n \in \mathscr{P}, m<n, \varepsilon=1 / 2$ is related to $F$ as in 2.1. Lip $D^{2} F<\infty, a \in \mathbf{R}^{m}, 0<r<\infty$, and $u, v \in \mathbf{W}^{1,2}\left(\mathbf{U}(a, r), \mathbf{R}^{n-m}\right)$ with $u-v \in \mathbf{W}_{0}^{1,2}\left(\mathbf{U}(a, r), \mathbf{R}^{n-m}\right)$.

Then for every affine function $P: \mathbf{R}^{m} \rightarrow \mathbf{R}^{n-m}$

$$
\begin{aligned}
r^{-1-m}|v-u|_{1 ; a, r} \leq \Gamma r^{-m} & \left(\left|L_{F}(v)-L_{F}(u)\right|_{-1,1 ; a, r}\right. \\
& \left.+\operatorname{Lip}\left(D^{2} F\right)\left(|\mathbf{D}(u-P)|_{2 ; a, r}+|\mathbf{D}(v-P)|_{2 ; a, r}\right)^{2}\right)
\end{aligned}
$$

where $\Gamma=\Gamma \overline{2.13}(n, 1 / 2 /, 3 / 2)$.

Proof. Let $\Lambda=\operatorname{Lip} D^{2} F$, choose $\sigma \in \operatorname{Hom}\left(\mathbf{R}^{m}, \mathbf{R}^{n-m}\right)$ such that $D P(x)=\sigma$ for $x \in \mathbf{R}^{m}$, and define $T=L_{F}(v)-L_{F}(u)$, the $\mathscr{L}^{m}\llcorner\mathbf{U}(a, r)$ measurable function $A: \mathbf{U}(a, r) \rightarrow \bigodot^{2} \operatorname{Hom}\left(\mathbf{R}^{m}, \mathbf{R}^{n-m}\right)$ by

$$
A(x)=\int_{0}^{1} D^{2} F(t \mathbf{D} v(x)+(1-t) \mathbf{D} u(x))-D^{2} F(\sigma) \mathrm{d} \mathscr{L}^{1} t
$$

whenever $x \in \mathbf{U}(a, r)$, and $S \in \mathscr{D}^{\prime}\left(\mathbf{U}(a, r), \mathbf{R}^{n-m}\right)$ by

$$
S(\theta)=\int_{\mathbf{U}(a, r)}\langle D \theta(x) \odot \mathbf{D}(v-u)(x), A(x)\rangle \mathrm{d} \mathscr{L}^{m} x+T(\theta)
$$


whenever $\theta \in \mathscr{D}\left(\mathbf{U}(a, r), \mathbf{R}^{n-m}\right)$. One computes

$$
\begin{aligned}
& D F(\mathbf{D} v(x))-D F(\mathbf{D} u(x)) \\
& \quad=\left\langle\mathbf{D}(v-u)(x), \int_{0}^{1} D D F(t \mathbf{D} v(x)+(1-t) \mathbf{D} u(x)) \mathrm{d} \mathscr{L}^{1} t\right\rangle
\end{aligned}
$$

for $\mathscr{L}^{n}$ almost all $x \in \mathbf{U}(a, r)$ and infers

$$
S(\theta)=-\int_{\mathbf{U}(a, r)}\left\langle D \theta(x) \odot \mathbf{D}(v-u)(x), D^{2} F(\sigma)\right\rangle \mathrm{d} \mathscr{L}^{m} x
$$

whenever $\theta \in \mathscr{D}\left(\mathbf{U}(a, r), \mathbf{R}^{n-m}\right)$, hence by 2.13 with $\Phi$ replaced by $D^{2} F(\sigma)$

$$
r^{-1-m}|v-u|_{1 ; a, r} \leq \Gamma r^{-m}|S|_{-1,1 ; a, r}
$$

It remains to estimate $|S|_{-1,1 ; a, r}$. By use of the definition of $S$ one estimates

$$
\begin{aligned}
\|A(x)\| & \leq \int_{0}^{1}\left\|D^{2} F(t \mathbf{D} v(x)+(1-t) \mathbf{D} u(x))-D^{2} F(t \sigma+(1-t) \sigma)\right\| \mathrm{d} \mathscr{L}^{1} t \\
& \leq \Lambda \int_{0}^{1} t|\mathbf{D}(v-P)(x)|+(1-t)|\mathbf{D}(u-P)(x)| \mathrm{d} \mathscr{L}^{1} t \\
& =\Lambda(|\mathbf{D}(v-P)(x)|+|\mathbf{D}(u-P)(x)|) / 2
\end{aligned}
$$

for $\mathscr{L}^{m}$ almost all $x \in \mathbf{U}(a, r)$. Finally,

$$
|S|_{-1,1 ; a, r} \leq|T|_{-1,1 ; a, r}+\Lambda / 2 \int_{\mathbf{U}(a, r)}(|\mathbf{D}(u-P)(x)|+|\mathbf{D}(v-P)(x)|)^{2} \mathrm{~d} \mathscr{L}^{m} x .
$$

2.16. Whenever $m, n \in \mathscr{P}, m<n, U$ is an open subset of $\mathbf{R}^{m}, a \in U$, and $T \in$ $\mathscr{D}^{\prime}\left(U, \mathbf{R}^{n-m}\right)$ there exists at most one constant distribution $T_{a} \in \mathscr{D}^{\prime}\left(U, \mathbf{R}^{n-m}\right)$ such that

$$
\lim _{r \rightarrow 0+} r^{-m-1}\left|T-T_{a}\right|_{-1,1 ; a, r}=0,
$$

see the last paragraph of the proof of 2.12

2.17 Lemma. Suppose $m, n \in \mathscr{P}, m<n$.

Then there exists a positive, finite number $\varepsilon$ with the following property.

If $F$ is related to $\varepsilon$ as in [2.1], Lip $D^{2} F<\infty, U$ is an open subset of $\mathbf{R}^{m}$, $u: U \rightarrow \mathbf{R}^{n-m}$ is weakly differentiable, $A_{1}$ denotes the set of all $a \in U$ such that

$$
\limsup _{r \rightarrow 0+} r^{-m-1}\left|L_{F}(u)\right|_{-1,1 ; a, r}<\infty,
$$

$A_{2}$ denotes the set of all $a \in U$ such that there exists a (unique, see [2.16) constant distribution $T_{a} \in \mathscr{D}^{\prime}\left(U, \mathbf{R}^{n-m}\right)$ such that

$$
\lim _{r \rightarrow 0+} r^{-m-1}\left|L_{F}(u)-T_{a}\right|_{-1,1 ; a, r}=0
$$

$B_{1}$ denotes the set of all $b \in \operatorname{dmn} \mathbf{D} u$ such that

$$
\limsup _{r \rightarrow 0+} r^{-m-1} \int_{\mathbf{U}(b, r)}|\mathbf{D} u(x)-\mathbf{D} u(b)|^{2} \mathrm{~d} \mathscr{L}^{m} x<\infty,
$$

and $B_{2}$ denotes the set of all $b \in \mathrm{dmn} \mathbf{D} u$ such that

$$
\lim _{r \rightarrow 0+} r^{-m-1} \int_{\mathbf{U}(b, r)}|\mathbf{D} u(x)-\mathbf{D} u(b)|^{2} \mathrm{~d} \mathscr{L}^{m} x=0,
$$

then the following two statements hold: 
(1) For $\mathscr{L}^{m}$ almost all $a \in A_{1} \cap B_{1}$ there exists a polynomial function $Q_{a}$ : $\mathbf{R}^{m} \rightarrow \mathbf{R}^{n-m}$ of degree at most 2 such that

$$
\lim _{r \rightarrow 0+} r^{-2-m}\left|u-Q_{a}\right|_{1 ; a, r}=0 .
$$

(2) If $a \in A_{2} \cap B_{2}$ satisfies the conclusion of (1) with $Q_{a}$ then

$$
T_{a}(\theta)=\int_{U} \theta(x) \bullet\left\langle D^{2} Q_{a}(a), C_{F}\left(D Q_{a}(a)\right)\right\rangle \mathrm{d} \mathscr{L}^{m} x
$$

for $\theta \in \mathscr{D}\left(U, \mathbf{R}^{n-m}\right)$ where $C_{F}$ is defined as in 2.1 .

Proof. Let

$$
\varepsilon=\inf \left\{1 / 2,2_{2.10}(m, n, 1,2), q 2.5(n, 2,2)\right\} .
$$

Suppose $F$ and $u$ satisfy the hypotheses with $\varepsilon$. Abbreviate $\Lambda=\operatorname{Lip} D^{2} F$ and $T=L_{F}(u)$. Fix $a \in A_{1} \cap B_{1}$ and $0<R<\infty$ such that $\mathbf{B}(a, R) \subset U$ and $u \mid \mathbf{U}(a, R) \in \mathbf{W}^{1,2}\left(\mathbf{U}(a, R), \mathbf{R}^{n-m}\right)$.

To prove part (11), the criterion 2.10 will be verified with $q=2, j=0$. Using the direct method of the calculus of variation, see e.g. Giu03, Theorems 4.5,6, Remark 4.1], one constructs for $0<r<R$ functions $v_{r} \in \mathbf{W}^{1,2}\left(\mathbf{U}(a, r), \mathbf{R}^{n-m}\right)$ such that

$$
v_{r}-u \in \mathbf{W}_{0}^{1,2}\left(\mathbf{U}(a, r), \mathbf{R}^{n-m}\right), \quad L_{F}\left(v_{r}\right)=0 .
$$

By 2.15 one estimates

$$
\begin{aligned}
& r^{-1-m}\left|v_{r}-u\right|_{1 ; a, r} \\
& \quad \leq \Delta_{1} r^{-m}\left(|T|_{-1,1 ; a, r}+\Lambda\left(|\mathbf{D}(u-\mathbf{D} u(a))|_{2 ; a, r}+\left|\mathbf{D}\left(v_{r}-\mathbf{D} u(a)\right)\right|_{2 ; a, r}\right)^{2}\right) .
\end{aligned}
$$

with $\Delta_{1}=\Gamma 2.15(n)$. By 2.14 with $c=1 / 2, M=2$ one infers

$$
\left|\mathbf{D}\left(v_{r}-u\right)\right|_{2 ; a, r} \leq 4|\mathbf{D}(u-\mathbf{D} u(a))|_{2 ; a, r},
$$

hence

$$
r^{-1-m}\left|v_{r}-u\right|_{1 ; a, r} \leq \Delta_{1} r^{-m}\left(|T|_{-1,1 ; a, r}+\Lambda\left(6|\mathbf{D}(u-\mathbf{D} u(a))|_{2 ; a, r}\right)^{2}\right) .
$$

Since $a \in A_{1} \cap B_{1}$, this implies

$$
\limsup _{r \rightarrow 0+} r^{-2-m}\left|v_{r}-u\right|_{1 ; a, r}<\infty .
$$

Therefore part (11) follows from 2.10.

To prove part (2), assume now additionally that the assumptions of (2) are valid for $a$, i.e. $a \in A_{2} \cap B_{2}$ and $Q_{a}$ satisfies the conclusion of (1). Choose $y \in \mathbf{R}^{n-m}$ such that

$$
T_{a}(\theta)=\int_{U} \theta(x) \bullet y \mathrm{~d} \mathscr{L}^{m} x \quad \text { for } \theta \in \mathscr{D}\left(U, \mathbf{R}^{n-m}\right) .
$$

Using the direct method of the calculus of variation as before, one constructs for $0<r<R$ functions $w_{r} \in \mathbf{W}^{1,2}\left(\mathbf{U}(a, r), \mathbf{R}^{n-m}\right)$ such that

$$
\begin{gathered}
w_{r}-u \in \mathbf{W}_{0}^{1,2}\left(\mathbf{U}(a, r), \mathbf{R}^{n-m}\right), \\
L_{F}\left(w_{r}\right)(\theta)=\int_{\mathbf{U}(a, r)} \theta(x) \bullet y \mathrm{~d} \mathscr{L}^{m} x \quad \text { whenever } \theta \in \mathscr{D}\left(\mathbf{U}(a, r), \mathbf{R}^{n-m}\right) .
\end{gathered}
$$


By 2.15 one estimates

$$
\begin{aligned}
r^{-1-m}\left|w_{r}-u\right|_{1 ; a, r} \leq & \Delta_{1} r^{-m}\left(\left|T-T_{a}\right|_{-1,1 ; a, r}\right. \\
& \left.+\Lambda\left(|\mathbf{D}(u-\mathbf{D} u(a))|_{2 ; a, r}+\left|\mathbf{D}\left(w_{r}-\mathbf{D} u(a)\right)\right|_{2 ; a, r}\right)^{2}\right) .
\end{aligned}
$$

Since, by Poincaré's inequality,

$$
\left|\int_{\mathbf{U}(a, r)} \theta(x) \bullet y \mathrm{~d} \mathscr{L}^{m} x\right| \leq|y| \Delta_{2} r^{1+m / 2}|D \theta|_{2 ; a, r}
$$

where $\Delta_{2}$ is a positive, finite number depending only on $n$, one infers from 2.14

$$
\left|\mathbf{D}\left(w_{r}-u\right)\right|_{2 ; a, r} \leq 4|\mathbf{D}(u-\mathbf{D} u(a))|_{2 ; a, r}+2 \Delta_{2}|y| r^{1+m / 2},
$$

hence

$$
\begin{aligned}
& r^{-1-m}\left|w_{r}-u\right|_{1 ; a, r} \\
& \quad \leq \Delta_{1} r^{-m}\left(\left|T-T_{a}\right|_{-1,1 ; a, r}+\Lambda\left(6|\mathbf{D}(u-\mathbf{D} u(a))|_{2 ; a, r}+2 \Delta_{2}|y| r^{1+m / 2}\right)^{2}\right) .
\end{aligned}
$$

Since $a \in A_{2} \cap B_{2}$, this implies

$$
\lim _{r \rightarrow 0+} r^{-2-m}\left|w_{r}-u\right|_{1 ; a, r}=0 .
$$

Therefore by the assumption on $Q_{a}$

$$
\lim _{r \rightarrow 0+} r^{-2-m}\left|w_{r}-Q_{a}\right|_{1 ; a, r}=0 .
$$

In order to estimate derivatives of $w_{r}-Q_{a}$, define $P: \mathbf{R}^{m} \rightarrow \mathbf{R}^{n-m}$ by $P(x)=Q_{a}(a)+\left\langle x-a, D Q_{a}(a)\right\rangle$ for $x \in \mathbf{R}^{m}, R=Q_{a}-P, S: \mathbf{R}^{m} \rightarrow \mathbf{R}^{n-m}$ by $S(x)=\frac{1}{2}\left\langle(x, x), D^{2} Q_{a}(a)\right\rangle$ for $x \in \mathbf{R}^{m}$ and note $r^{-2} R \circ \boldsymbol{\tau}_{a} \circ \boldsymbol{\mu}_{r}=S$ and

$$
r^{-2}\left(w_{r}-P\right) \circ \boldsymbol{\tau}_{a} \circ \boldsymbol{\mu}_{r}|\mathbf{U}(0,1) \rightarrow S| \mathbf{U}(0,1) \quad \text { in } \mathbf{L}_{1}\left(\mathbf{U}(0,1), \mathbf{R}^{n-m}\right)
$$

as $r \rightarrow 0+$. By 2.5

$$
r^{-m / 2}\left|\mathbf{D}^{2}\left(w_{r}-P\right)\right|_{2 ; a, r / 2} \leq \Delta_{3}\left(r^{-2-m}\left|w_{r}-P\right|_{1 ; a, r}+|y|\right)
$$

where $\Delta_{3}=\sup \left\{1, \boldsymbol{\alpha}(m)^{1 / 2}\right\} \Gamma[2.5(n, 2)$, hence

$$
\limsup _{r \rightarrow 0+} r^{-m / 2}\left|\mathbf{D}^{2}\left(w_{r}-P\right)\right|_{2 ; a, r / 2}<\infty .
$$

By Rellich's embedding theorem

$$
\begin{gathered}
r^{-2}\left(w_{r}-P\right) \circ \boldsymbol{\tau}_{a} \circ \boldsymbol{\mu}_{r}|\mathbf{U}(0,1 / 2) \rightarrow S| \mathbf{U}(0,1 / 2) \quad \text { in } \mathbf{W}^{1,2}\left(\mathbf{U}(0,1 / 2), \mathbf{R}^{n-m}\right), \\
r^{-2}\left(w_{r}-Q_{a}\right) \circ \boldsymbol{\tau}_{a} \circ \boldsymbol{\mu}_{r} \mid \mathbf{U}(0,1 / 2) \rightarrow 0 \quad \text { in } \mathbf{W}^{1,2}\left(\mathbf{U}(0,1 / 2), \mathbf{R}^{n-m}\right)
\end{gathered}
$$

as $r \rightarrow 0+$. This convergence implies

$$
\begin{gathered}
\left|r^{-m-1} \int_{\mathbf{U}(a, r / 2)}\left\langle(D \theta) \circ \boldsymbol{\mu}_{1 / r} \circ \boldsymbol{\tau}_{-a}(x), D F\left(\mathbf{D} w_{r}(x)\right)-D F\left(D Q_{a}(x)\right)\right\rangle \mathrm{d} \mathscr{L}^{m} x\right| \\
\leq r^{-m / 2-1}(\operatorname{Lip} D F)|D \theta|_{2 ; 0,1}\left|\mathbf{D}\left(w_{r}-Q_{a}\right)\right|_{2 ; a, r} \rightarrow 0 \quad \text { as } r \rightarrow 0+
\end{gathered}
$$


for $\theta \in \mathscr{D}\left(\mathbf{U}(0,1 / 2), \mathbf{R}^{n-m}\right)$. Therefore, noting

$$
\begin{gathered}
\int_{\mathbf{U}(0,1 / 2)} \theta(x) \bullet y \mathrm{~d} \mathscr{L}^{m} x=r^{-m} \int_{\mathbf{U}(a, r / 2)}\left(\theta \circ \boldsymbol{\mu}_{1 / r} \circ \boldsymbol{\tau}_{-a}\right)(x) \bullet y \mathrm{~d} \mathscr{L}^{m} x \\
=-r^{-m-1} \int_{\mathbf{U}(a, r / 2)}\left\langle(D \theta) \circ \boldsymbol{\mu}_{1 / r} \circ \boldsymbol{\tau}_{-a}(x), D F\left(\mathbf{D} w_{r}(x)\right)\right\rangle \mathrm{d} \mathscr{L}^{m} x
\end{gathered}
$$

for $\theta \in \mathscr{D}\left(\mathbf{U}(0,1 / 2), \mathbf{R}^{n-m}\right)$ and

$$
\begin{gathered}
-r^{-m-1} \int_{\mathbf{U}(a, r / 2)}\left\langle(D \theta) \circ \boldsymbol{\mu}_{1 / r} \circ \boldsymbol{\tau}_{-a}(x), D F\left(D Q_{a}(x)\right)\right\rangle \mathrm{d} \mathscr{L}^{m} x \\
=r^{-m} \int_{\mathbf{U}(a, r / 2)}\left(\theta \circ \boldsymbol{\mu}_{1 / r} \circ \boldsymbol{\tau}_{-a}\right)(x) \bullet\left\langle D^{2} Q_{a}(x), C_{F}\left(D Q_{a}(x)\right)\right\rangle \mathrm{d} \mathscr{L}^{m} x \\
\quad \rightarrow \int_{\mathbf{U}(0,1 / 2)} \theta(x) \bullet\left\langle D^{2} Q_{a}(a), C_{F}\left(D Q_{a}(a)\right)\right\rangle \mathrm{d} \mathscr{L}^{m} x \quad \text { as } r \rightarrow 0+,
\end{gathered}
$$

for $\theta \in \mathscr{D}\left(\mathbf{U}(0,1 / 2), \mathbf{R}^{n-m}\right)$, one infers

$$
y=\left\langle D^{2} Q_{a}(a), C_{F}\left(D Q_{a}(a)\right)\right\rangle,
$$

as asserted.

2.18 Remark. Clearly, by Rešetnyak [Reš68] or [Fed69, 4.5.9 (26) (II) (III)] for $\mathscr{L}^{m}$ almost all $a \in A_{1} \cap B_{1}$

$$
Q_{a}(a)=u(a), \quad D Q_{a}(a)=\mathbf{D} u(a)
$$

Also by Calderón and Zygmund [CZ61, Theorem 9] (see also [Zie89, 3.6-8]), there exists a sequence of functions $u_{i}: \mathbf{R}^{m} \rightarrow \mathbf{R}^{n-m}$ of class 2 such that

$$
\mathscr{L}^{m}\left(A_{1} \cap B_{1} \sim \bigcup_{i=1}^{\infty}\left\{a: D^{k} u_{i}(a)=D^{k} Q_{a}(a) \text { for } k \in\{0,1,2\}\right\}\right)=0 .
$$

2.19 Remark. In A.3 it will be shown $\mathscr{L}^{m}\left(A_{1} \sim A_{2}\right)=0$.

2.20 Lemma. Suppose $H$ is a Hilbert space with $\operatorname{dim} H=N<\infty, k, l \in$ $\mathscr{P} \cup\{0\}, l \geq k, \Phi: H \rightarrow \mathbf{R}$ is of class $l, a \in H, 0<\delta<\infty$, and

$$
s=\sup \left\{\left\|D^{k} \Phi(x)-D^{k} \Phi(a)\right\|: x \in \mathbf{B}(a, \delta)\right\} .
$$

Then there exists $F: H \rightarrow \mathbf{R}$ of class l such that

$$
\begin{gathered}
D^{i} F(x)=D^{i} \Phi(x) \quad \text { for } x \in \mathbf{B}(a, \delta / 2), i=0, \ldots, k, \\
\left\|D^{k} F(x)-D^{k} \Phi(a)\right\| \leq \Gamma s \quad \text { for } x \in H,
\end{gathered}
$$

$F \mid H \sim \mathbf{B}(a, \delta)$ is the restriction of a polynomial function of degree at most $k$ where $\Gamma$ is a positive, finite number depending only on $N$ and $k$.

Proof. Choosing $\varphi \in \mathscr{E}^{0}(\mathbf{R})$ with $0 \leq \varphi(t) \leq 1$ for $t \in \mathbf{R}$ and

$$
\{t:-\infty<t \leq 1 / 2\} \subset \operatorname{Int}\{t: \varphi(t)=1\}, \quad\{t: 1 \leq t<\infty\} \subset \operatorname{Int}\{t: \varphi(t)=0\}
$$

one defines $P: H \rightarrow \mathbf{R}, F: H \rightarrow \mathbf{R}$ by

$$
\begin{gathered}
P(x)=\sum_{i=0}^{k}\left\langle(x-a)^{i} / i !, D^{i} \Phi(a)\right\rangle, \\
F(x)=P(x)+\varphi(|x-a| / \delta)(\Phi(x)-P(x))
\end{gathered}
$$

for $x \in H$ and readily estimates $\left\|D^{k} F(x)-D^{k} \Phi(a)\right\|$ be means of Taylor's formula (cf. [Fed69, 3.1.11]). 


\section{An approximate second order structure for certain integral varifolds}

In this Section 3.6 which is Theorem 1 of the Introduction is proven. In order to do this a general lemma is established which states that the part of the varifold exhibiting a certain decay of its tilt-excess can be covered with some accuracy by suitable rotated graphs of Lipschitzian function having similar decay properties of their "tilt-excess". This is done by carefully combining the approximation by $\mathbf{Q}_{Q}\left(\mathbf{R}^{n-m}\right)$ valued functions of [Men09b, 4.8] with more basic differentiability results in Men09a]. The "tilt-excess" decay of the Lipschitzian functions is the nonintegral differentiability condition used in Section 2 to compensate for the use of the weak norm $|\cdot|_{-1,1 ; a, s}$ in the estimates which seems to be inavoidable, see 3.4

3.1 Lemma. Suppose $n, Q \in \mathscr{P}, 0<L<\infty, 1 \leq M<\infty, 0<\delta_{i} \leq 1$ for $i \in\{1,2,3\}$, and $0<\delta_{4} \leq 1 / 4$.

Then there exists a positive, finite number $\varepsilon$ with the following property.

If $m \in \mathscr{P}, m<n, 0<s<\infty, S=\operatorname{im} \mathbf{p}^{*}$,

$$
U=\left(\mathbf{R}^{m} \times \mathbf{R}^{n-m}\right) \cap\{(x, y): \operatorname{dist}((x, y), \mathbf{C}(S, 0, s, s))<2 s\},
$$

$V \in \mathbf{I V}_{m}(U),\|\delta V\|$ is a Radon measure,

$$
\begin{gathered}
\left(Q-1+\delta_{1}\right) \boldsymbol{\alpha}(m) s^{m} \leq\|V\|(\mathbf{C}(S, 0, s, s)) \leq\left(Q+1-\delta_{2}\right) \boldsymbol{\alpha}(m) s^{m}, \\
\|V\|\left(\mathbf{C}\left(S, 0, s, s+\delta_{4} s\right) \sim \mathbf{C}\left(S, 0, s, s-2 \delta_{4} s\right)\right) \leq\left(1-\delta_{3}\right) \boldsymbol{\alpha}(m) s^{m} \\
\|V\|(U) \leq M \boldsymbol{\alpha}(m) s^{m}
\end{gathered}
$$

$0<\delta \leq \varepsilon, B$ denotes the set of all $z \in \mathbf{C}(S, 0, s, s)$ with $\boldsymbol{\Theta}^{* m}(\|V\|, z)>0$ such that

$$
\begin{gathered}
\quad \text { either } \quad\|\delta V\| \mathbf{B}(z, t)>\delta\|V\|(\mathbf{B}(z, t))^{1-1 / m} \quad \text { for some } 0<t<2 s, \\
\text { or } \quad \int_{\mathbf{B}(z, t) \times \mathbf{G}(n, m)}\left|R_{\natural}-S_{\natural}\right| \mathrm{d} V(\xi, R)>\delta\|V\| \mathbf{B}(z, t) \quad \text { for some } 0<t<2 s,
\end{gathered}
$$

$A=\mathbf{C}(S, 0, s, s) \sim B, A(x)=A \cap\{z: \mathbf{p}(z)=x\}$ for $x \in \mathbf{R}^{m}, X_{1}$ is the set of all $x \in \mathbf{R}^{m} \cap \mathbf{B}(0, s)$ such that

$$
\sum_{z \in A(x)} \boldsymbol{\Theta}^{m}(\|V\|, z)=Q \quad \text { and } \quad \boldsymbol{\Theta}^{m}(\|V\|, z) \in \mathscr{P} \cup\{0\} \text { for } z \in A(x),
$$

$X_{2}$ is the set of all $x \in \mathbf{R}^{m} \cap \mathbf{B}(0, s)$ such that

$$
\sum_{z \in A(x)} \Theta^{m}(\|V\|, z) \leq Q-1 \quad \text { and } \quad \Theta^{m}(\|V\|, z) \in \mathscr{P} \cup\{0\} \text { for } z \in A(x),
$$

$N=\mathbf{R}^{m} \cap \mathbf{B}(0, s) \sim\left(X_{1} \cup X_{2}\right)$, and $f: X_{1} \rightarrow \mathbf{Q}_{Q}\left(\mathbf{R}^{n-m}\right)$ is characterised by the requirement

$$
\boldsymbol{\Theta}^{m}(\|V\|, z)=\boldsymbol{\Theta}^{0}(\|f(x)\|, \mathbf{q}(z)) \quad \text { whenever } x \in X_{1} \text { and } z \in A(x),
$$

then the following seven statements hold:

(1) $X_{1}$ and $X_{2}$ are universally measurable, and $\mathscr{L}^{m}(N)=0$.

(2) $A$ and $B$ are Borel sets and

$$
\mathbf{q}[A \cap \operatorname{spt}\|V\|] \subset \mathbf{B}\left(0, s-\delta_{4} s\right) .
$$


(3) $\mathbf{p}\left[A \cap\left\{z: \mathbf{\Theta}^{m}(\|V\|, z)=Q\right\}\right] \subset X_{1}$.

(4) The function $f$ is Lipschitzian with $\operatorname{Lip} f \leq L$.

(5) For $\mathscr{L}^{m}$ almost all $x \in X_{1}$ the following is true:

(a) The function $f$ is approximately strongly affinely approximable at $x$.

(b) If $(x, y) \in \operatorname{graph}_{Q} f$ then

$$
\operatorname{Tan}^{m}(\|V\|,(x, y))=\operatorname{Tan}\left(\operatorname{graph}_{Q} \text { ap } A f(x),(x, y)\right) \in \mathbf{G}(n, m) .
$$

(6) If $a \in A, \mathbf{\Theta}^{m}(\|V\|, a)=Q, 0<t \leq s-|\mathbf{p}(a)|,|\mathbf{q}(a)|+\delta_{4} t \leq s$, and

$$
\begin{aligned}
B_{a, t} & =\mathbf{C}\left(S, a, t, \delta_{4} t\right) \cap B, \\
C_{a, t} & =\mathbf{B}(\mathbf{p}(a), t) \sim\left(X_{1} \sim \mathbf{p}\left[B_{a, t}\right]\right), \\
D_{a, t} & =\mathbf{C}\left(S, a, t, \delta_{4} t\right) \cap \mathbf{p}^{-1}\left[C_{a, t}\right],
\end{aligned}
$$

then $B_{a, t}$ is a Borel set, $C_{a, t}$ and $D_{a, t}$ are universally measurable and

$$
\mathscr{L}^{m}\left(C_{a, t}\right)+\|V\|\left(D_{a, t}\right) \leq \Gamma_{[\underline{6}}\|V\|\left(B_{a, t}\right)
$$

with $\Gamma_{[6]}=3+2 Q+(12 Q+6) 5^{m}$.

(7) If $a, t, C_{a, t}, D_{a, t}$ are as in (6), $g: \mathbf{R}^{m} \rightarrow \mathbf{R}^{n-m}$, Lip $g<\infty, g \mid X_{1}=$ $\boldsymbol{\eta}_{Q} \circ f, \tau \in \operatorname{Hom}\left(\mathbf{R}^{m}, \mathbf{R}^{n-m}\right), \theta \in \mathscr{D}\left(\mathbf{R}^{m}, \mathbf{R}^{n-m}\right), \eta \in \mathscr{D}^{0}\left(\mathbf{R}^{n-m}\right)$,

$$
\operatorname{spt} \theta \subset \mathbf{U}(\mathbf{p}(a), t), \quad 0 \leq \eta(y) \leq 1 \quad \text { for } y \in \mathbf{R}^{n-m},
$$

$$
\operatorname{spt} \eta \subset \mathbf{U}\left(\mathbf{q}(a), \delta_{4} t\right), \quad \mathbf{B}\left(\mathbf{q}(a), \delta_{4} t / 2\right) \subset \operatorname{Int}\left(\mathbf{R}^{n-m} \cap\{y: \eta(y)=1\}\right),
$$

and $\Psi^{\S}$ denotes the nonparametric integrand associated to the area integrand $\Psi$, then

$$
\begin{aligned}
\mid Q \int\langle D & \left.\theta(x), D \Psi_{0}^{\S}(D g(x))\right\rangle \mathrm{d} \mathscr{L}^{m} x-(\delta V)\left((\eta \circ \mathbf{q}) \cdot\left(\mathbf{q}^{*} \circ \theta \circ \mathbf{p}\right)\right) \mid \\
\leq & \gamma_{1} Q m^{1 / 2} \operatorname{Lip} g \int_{C_{a, t}}|D \theta| \mathrm{d} \mathscr{L}^{m} \\
\quad & +\gamma_{2} \int_{E_{a, t} \sim C_{a, t}}|D \theta(x)||\operatorname{ap} A f(x)(+)(-\tau)|^{2} \mathrm{~d} \mathscr{L}^{m} x \\
\quad & +m^{1 / 2} \int_{D_{a, t}}\left|D\left((\eta \circ \mathbf{q}) \cdot\left(\mathbf{q}^{*} \circ \theta \circ \mathbf{p}\right)\right)\right| \mathrm{d}\|V\|
\end{aligned}
$$

where

$$
\begin{gathered}
\gamma_{1}=\sup \left\|D^{2} \Psi_{0}^{\S}\right\|\left[\mathbf{B}\left(0, m^{1 / 2} \operatorname{Lip} g\right)\right], \\
\gamma_{2}=\operatorname{Lip}\left(D^{2} \Psi_{0}^{\S} \mid \mathbf{B}\left(0, m^{1 / 2}(L+2\|\tau\|)\right)\right), \\
E_{a, t}=\mathbf{B}(\mathbf{p}(a), t) \cap X_{1} \cap\left\{x: \Theta^{0}(\|f(x)\|, g(x)) \neq Q\right\} .
\end{gathered}
$$

Proof. This follows from [Men09b, 4.8, 10]; in fact the statements (11)-(5) are those in Men09b, 4.8] with $r, h, T$ replaced by $s, s, S$ and [Men09b, 4.10] shows that the additional conditions $a \in A$ and $\Theta^{m}(\|V\|, a)=$,$Q in (6) (7) can be$ arranged to imply

$$
\begin{gathered}
\operatorname{graph}_{Q} f \mid \mathbf{B}(\mathbf{p}(a), t) \subset \mathbf{C}\left(S, a, t, \delta_{4} t / 2\right), \\
\|V\|\left(\mathbf{C}\left(S, a, t, \delta_{4} t\right)\right) \geq(Q-1 / 4) \boldsymbol{\alpha}(m) t^{m},
\end{gathered}
$$

hence (6) (7) are consequences of [Men09b, 4.8 (6) (7) (9)]. 
3.2. The following situation will be studied: $m, n \in \mathscr{P}, m<n, 1 \leq p \leq \infty, U$ is an open subset of $\mathbf{R}^{n}, V \in \mathbf{V}_{m}(U),\|\delta V\|$ is a Radon measure and, if $p>1$,

$$
\begin{gathered}
(\delta V)(g)=-\int g(z) \bullet \mathbf{h}(V ; z) \mathrm{d}\|V\|(z) \quad \text { whenever } g \in \mathscr{D}\left(U, \mathbf{R}^{n}\right), \\
\mathbf{h}(V ; \cdot) \in \mathbf{L}_{p}\left(\|V\|\left\llcorner K, \mathbf{R}^{n}\right) \quad \text { whenever } K \text { is a compact subset of } U .\right.
\end{gathered}
$$

If $p<\infty$ then the measure $\psi$ is defined by

$$
\psi=\|\delta V\| \quad \text { if } p=1, \quad \psi=|\mathbf{h}(V ; \cdot)|^{p}\|V\| \quad \text { if } p>1 \text {. }
$$

3.3 Lemma. Suppose $m, n \in \mathscr{P}, m<n, 1 \leq p \leq m, 1 \leq q<\infty, 0<\alpha \leq 1$, $\alpha q(m-p) \leq m p, 0<L<\infty, V \in \mathbf{I V}_{m}(U), \psi$ is related to $p$ and $V$ as in 3.2, and $P$ is the set of all $a \in U$ such that $\operatorname{Tan}^{m}(\|V\|, a) \in \mathbf{G}(n, m)$ and

$$
\limsup _{s \rightarrow 0+} s^{-\alpha-m / q}\left(\int_{\mathbf{B}(a, s) \times \mathbf{G}(n, m)}\left|S_{\natural}-\operatorname{Tan}^{m}(\|V\|, a)_{\natural}\right|^{q} \mathrm{~d} V(z, S)\right)^{1 / q}<\infty .
$$

Then there exists a countable, disjointed family $H$ of $\|V\|$ measurable subsets of $P$ such that $\|V\|(P \sim \bigcup H)=0$ and for each $Z \in H$ there exists a nonempty open subset $O$ of $\mathbf{O}^{*}(n, m)$ such that for each $\pi_{1} \in O$ there exist

$$
\begin{gathered}
g: \mathbf{R}^{m} \rightarrow \mathbf{R}^{n-m}, \quad G: \mathbf{R}^{m} \rightarrow \mathbf{R}^{n}, \quad K \subset \mathbf{R}^{m}, \quad Q \in \mathscr{P}, \\
\pi_{2} \in \mathbf{O}^{*}(n, n-m), \quad T \in \mathscr{D}^{\prime}\left(\mathbf{R}^{m}, \mathbf{R}^{n-m}\right)
\end{gathered}
$$

with the following six properties:

(1) $\pi_{2} \circ \pi_{1}^{*}=0, G=\pi_{1}^{*}+\pi_{2}^{*} \circ g$, and $G[K]=Z$.

(2) $\operatorname{Lip} g \leq L$.

(3) $K$ is an $\mathscr{L}^{m}$ measurable subset of $\operatorname{dmn} D g$.

(4) $\int\left\langle D \theta(x), D \Psi_{0}^{\S}(D g(x))\right\rangle \mathrm{d} \mathscr{L}^{m} x=T(\theta)$ for $\theta \in \mathscr{D}\left(\mathbf{R}^{m}, \mathbf{R}^{n-m}\right)$ where $\Psi$ denotes the area integrand.

(5) Whenever $x \in K$ there holds with $z=G(x)$ and $R=\operatorname{Tan}^{m}(\|V\|, z)$

$$
\begin{gathered}
\boldsymbol{\Theta}^{m}(\|V\|, z)=Q, \quad \operatorname{im} D G(x)=R \\
\limsup _{s \rightarrow 0+} s^{-\beta-m / r}\left(\int_{\mathbf{B}(x, s)}|D g(\zeta)-D g(x)|^{r} \mathrm{~d} \mathscr{L}^{m} \zeta\right)^{1 / r} \\
\leq 2 m^{1 / 2} \limsup _{s \rightarrow 0+} s^{-\beta-m / r}\left(\int_{\mathbf{B}(z, s) \times \mathbf{G}(n, m)}\left|S_{\natural}-R_{\natural}\right|^{r} \mathrm{~d} V(\xi, S)\right)^{1 / r}
\end{gathered}
$$

whenever $0<\beta \leq 1,1 \leq r<\infty$ and $\beta r \leq \alpha q$.

(6) Whenever $x \in K$ there holds

$$
\lim _{s \rightarrow 0+} s^{-m-1}\left|T-T_{x}\right|_{-1,1 ; x, s}=0
$$

where $T_{x} \in \mathscr{D}^{\prime}\left(\mathbf{R}^{m}, \mathbf{R}^{n-m}\right)$ is defined by

$$
T_{x}(\theta)=-\int \Psi_{0}^{\S}(D g(x)) \mathbf{h}(V ; G(x)) \bullet\left(\pi_{2}^{*} \circ \theta\right)(\zeta) \mathrm{d} \mathscr{L}^{m} \zeta
$$

whenever $\theta \in \mathscr{D}\left(\mathbf{R}^{m}, \mathbf{R}^{n-m}\right)$. 
Proof. First, observe that if some $\|V\|$ measurable set $Z$ has the properties listed in the conclusion so does every $\|V\|$ measurable subset of $Z$. Therefore, in order to prove the assertion, it is enough to show that for $\|V\|$ almost all $a \in P$ there exists a $\|V\|$ measurable set $Z$ having the stated properties and additionally satisfies $\Theta^{* m}(\|V\|\llcorner Z, a)>0$; in fact one can then take a maximal, disjointed family $H$ of such $Z$ (hence $\|V\|(Z)>0$ ) and note $H$ is countable and $\Theta^{m}\left(\|V\|\llcorner\bigcup H, a)=0\right.$ for $\mathscr{H}^{m}$ almost all $a \in U \sim \bigcup H$ by [Fed69, 2.10.19(4)] so that $\|V\|(P \sim \bigcup H)>0$ would contradict the maximality of $H$.

Define $P^{\prime}$ to be the set of all $z \in U$ such that $\operatorname{Tan}^{m}(\|V\|, z) \in \mathbf{G}(n, m)$ and

$$
\lim _{t \rightarrow 0+} t^{-1 / 2-m / 2}\left(\int_{\mathbf{B}(z, t) \times \mathbf{G}(n, m)}\left|S_{\natural}-\operatorname{Tan}^{m}(\|V\|, z)_{\natural}\right|^{2} \mathrm{~d} V(\xi, S)\right)^{1 / 2}=0 .
$$

By Brakke Bra78, 5.7,5] or [Men09b, 8.6] there holds $\|V\|\left(U \sim P^{\prime}\right)=0$. Therefore one may assume $\alpha q \geq 1$ possibly replacing $\alpha, q$ by $1 / 2,2$ if $\alpha q<1$. Assume further $L \leq 1 / 8$ and suppose $Q \in \mathscr{P}$. The remaining assertion will be shown to hold for $\|V\|$ almost all $a \in P$ with $\Theta^{m}(\|V\|, a)=Q$. For this purpose define

$$
\begin{gathered}
\delta_{1}=\delta_{2}=\delta_{3}=1 / 2, \quad \delta_{4}=1 / 4, \quad M=5^{m} Q, \\
\varepsilon=\inf \left\{\text { q3.1 }\left(n, Q, L, M, \delta_{1}, \delta_{2}, \delta_{3}, \delta_{4}\right),(2 \gamma(m))^{-1}\right\},
\end{gathered}
$$

and $R: U \cap\left\{z: \operatorname{Tan}^{m}(\|V\|, z) \in \mathbf{G}(n, m)\right\} \rightarrow \operatorname{Hom}\left(\mathbf{R}^{n}, \mathbf{R}^{n}\right)$ by

$$
R(z)=\operatorname{Tan}^{m}(\|V\|, z)_{\natural} \quad \text { whenever } z \in U \text { with } \operatorname{Tan}^{m}(\|V\|, z) \in \mathbf{G}(n, m) .
$$

For $i \in \mathscr{P}$ let $C_{i}$ denote the set of all $z \in \operatorname{spt}\|V\|$ such that either $\mathbf{B}(z, 1 / i) \not \subset U$ or

$$
\|\delta V\| \mathbf{B}(z, t)>(2 \varepsilon / 3)\|V\|(\mathbf{B}(z, t))^{1-1 / m} \quad \text { for some } 0<t<1 / i,
$$

let $D_{i}(w)$ for $w \in \operatorname{dmn} R$ denote the set of all $z \in U$ such that either $\mathbf{B}(z, 1 / i) \not \subset$ $U$ or

$$
\int_{\mathbf{B}(z, t)}|R(\xi)-R(w)|^{q} \mathrm{~d}\|V\| \xi>(\varepsilon / 3)^{q}\|V\| \mathbf{B}(z, t) \quad \text { for some } 0<t<1 / i
$$

and define $X_{i}$ for $i \in \mathscr{P}$ by

$$
\begin{gathered}
X_{i}=U \cap\left\{z: \Theta^{m^{2} /(m-p)}\left(\|V\|\left\llcorner C_{i}, z\right)=0\right\} \quad \text { if } p<m,\right. \\
X_{i}=U \sim \operatorname{Clos} C_{i} \quad \text { if } p=m,
\end{gathered}
$$

as well as $Y_{i}$ for $i \in \mathscr{P}$ by

$$
Y_{i}=(\operatorname{dmn} R) \cap\left\{w: \mathbf{\Theta}^{m+\alpha q}\left(\|V\|\left\llcorner D_{i}(w), w\right)=0\right\} .\right.
$$

Since $C_{i+1} \subset C_{i}$ and $D_{i+1}(w) \subset D_{i}(w)$ for $w \in \operatorname{dmn} R$, one notes $X_{i} \subset X_{i+1}$ and $Y_{i} \subset Y_{i+1}$ for $i \in \mathscr{P}$. $X_{i}$ are Borel sets. $Y_{i}$ are $\|V\|$ measurable sets by Men09a, 3.7 (ii)]. $P$ is $\|V\|$ measurable by [Men09a, 3.7]. Moreover,

$$
\|V\|\left(U \sim \bigcup\left\{X_{i}: i \in \mathscr{P}\right\}\right)=0, \quad\|V\|\left(P \sim \bigcup\left\{Y_{i}: i \in \mathscr{P}\right\}\right)=0
$$

by [Men09a, 2.5, 9, 10, 3.7 (ii)]. 
Define a measure $\mu$ on $U$ such that $\mu+|\mathbf{h}(V ; \cdot)|\|V\|=\|\delta V\|$ and $J=$ $P \cap\left\{z: \Theta^{m}(\|V\|, z)=Q\right\}$. The remaining assertion will be shown at a point $a$ such that for some $i \in \mathscr{P}$

$$
\begin{gathered}
a \in X_{i} \cap Y_{i} \cap(\operatorname{dmn} R), \quad \mathbf{B}(a, 4 / i) \subset U, \\
\boldsymbol{\Theta}^{m}(\|V\|, a)=Q, \quad \boldsymbol{\Theta}^{m}\left(\|V\|\left\llcorner U \sim\left(J \cap X_{i} \cap Y_{i}\right), a\right)=0,\right.
\end{gathered}
$$

$R$ is approximately continuous at $a$ with respect to $\|V\|$.

These conditions are satisfied by $\|V\|$ almost all $a \in J$ by the preceding remarks and [Fed69, 2.9.11,13]. Fix such $a$ and $i$, choose $0<\kappa \leq 1 / 2$ such that $(1+$ $\kappa)^{m} Q<Q+1 / 2$, and define $\lambda=\left(1+\kappa^{2}\right)^{-1 / 2}$ and $\delta=(1-\lambda) / 2$. Noting for $S \in \mathbf{G}(n, m)$ with $\left|S_{\natural}-R(a)\right|<\delta$ and $0<s<\infty$

$$
\begin{gathered}
\mathbf{R}^{n} \cap\left\{z:\left|S_{\natural}(z-a)\right| \leq \lambda|z-a|\right\} \subset \mathbf{R}^{n} \cap\{z:|R(a)(z-a)| \leq(\lambda+\delta)|z-a|\}, \\
\mathbf{C}(S, a, s) \cap\left\{z:\left|S_{\natural}(z-a)\right|>\lambda|z-a|\right\} \subset \mathbf{C}(S, a, s, \kappa s) \subset \mathbf{B}(a,(1+\kappa) s), \\
0<\lambda+\delta<1, \quad \boldsymbol{\Theta}^{m}(\|V\|\llcorner\{z:|R(a)(z-a)| \leq(\lambda+\delta)|z-a|\}, a)=0
\end{gathered}
$$

by [Fed69, 3.2.16], one infers the existence of $0<s<(2 i)^{-1}$ such that

$$
\begin{gathered}
(Q-1 / 2) \boldsymbol{\alpha}(m) s^{m} \leq\|V\|(\mathbf{C}(S, a, s, s)) \leq(Q+1 / 2) \boldsymbol{\alpha}(m) s^{m}, \\
\|V\|(\mathbf{C}(S, a, s, 5 s / 4) \sim \mathbf{C}(S, a, s, s / 2)) \leq(1 / 2) \boldsymbol{\alpha}(m) s^{m}, \\
\|V\|\left(\mathbf{R}^{n} \cap\{z: \operatorname{dist}(z, \mathbf{C}(S, a, s, s))<2 s\}\right) \leq\|V\| \mathbf{B}(a, 4 s) \leq M \boldsymbol{\alpha}(m) s^{m},
\end{gathered}
$$

whenever $S \in \mathbf{G}(n, m)$ with $\left|S_{\natural}-R(a)\right|<\delta$.

Define $A$ to be the set of all $z \in \mathbf{U}(a, s) \cap$ spt $\|V\|$ such that

$$
\begin{gathered}
\|\delta V\| \mathbf{B}(z, t) \leq(2 \varepsilon / 3)\|V\|(\mathbf{B}(z, t))^{1-1 / m}, \\
\int_{\mathbf{B}(z, t)}|R(\xi)-R(a)| \mathrm{d}\|V\| \xi \leq(2 \varepsilon / 3)\|V\| \mathbf{B}(z, t)
\end{gathered}
$$

whenever $0<t<2 s$,

$$
\begin{gathered}
O=\mathbf{O}^{*}(n, m) \cap\left\{\pi:\left|\pi^{*} \circ \pi-R(a)\right|<\inf \{\delta, \varepsilon / 3\}\right\}, \\
W=\mathbf{U}(a, s) \cap X_{i} \cap Y_{i} \cap\{w:|R(w)-R(a)| \leq \varepsilon / 3\}, \quad Z=W \cap A \cap J \sim N
\end{gathered}
$$

where $N$ is the set of all $w \in W$ such that one of the following three conditions is violated

$$
w \in P^{\prime}, \quad \Theta^{m}(\mu, w)=0, \quad \lim _{t \rightarrow 0+} t^{-m} \int_{\mathbf{B}(w, t)}|\mathbf{h}(V ; \xi)-\mathbf{h}(V ; w)| \mathrm{d}\|V\| \xi=0 .
$$

Note $\|V\|(N)=0$ by [Fed69, 2.9.10,11].

Now, fix $\pi_{1} \in O, S=\operatorname{im} \pi_{1}^{*}$ and choose $\pi_{2} \in \mathbf{O}^{*}(n, n-m)$ with $\pi_{2} \circ \pi_{1}^{*}=0$. The proof will be concluded by showing $\Theta^{m}(\|V\|\llcorner Z, a)=Q$ and constructing $g, G, K$ and $T$ with the asserted properties. For this purpose assume $a=0$ and $\pi_{1}=\mathbf{p}$ and $\pi_{2}=\mathbf{q}$ using isometries and identifying $\mathbf{R}^{n} \simeq \mathbf{R}^{m} \times \mathbf{R}^{n-m}$. Define

$$
u(w)=(s-|w-a|) / 2 \text { for } w \in W
$$

and note $u(w)>0$. Moreover, define $B, f$ as in 3.1 with $\delta$ replaced by $\varepsilon$ and whenever $w \in W$ and $0<t \leq u(w)$ define $B_{w, t}, C_{w, t}$ and $D_{w, t}$ as in 
3.1(6) (7) with additionally $a, s$ replaced by $w$, $t$. Since $\left|S_{\natural}-R(a)\right| \leq \varepsilon / 3$ and $Z \subset A \cap\left\{z: \Theta^{m}(\|V\|, z)=Q\right\}$, one infers from 3.1(3) that $Z \subset \operatorname{graph}_{Q} f$ and

$$
\Theta^{0}(\|f(\mathbf{p}(z))\|, \mathbf{q}(z))=Q, \quad\left(\mathbf{p}^{*}+\mathbf{q}^{*} \circ \boldsymbol{\eta}_{Q} \circ f\right)(\mathbf{p}(z))=z
$$

whenever $z \in Z$. Using Kirszbraun's theorem (cf. Fed69, 2.10.43]) one extends $\boldsymbol{\eta}_{Q} \circ f$ to a function $g: \mathbf{R}^{m} \rightarrow \mathbf{R}^{n-m}$ such that

$$
\operatorname{Lip} g=\operatorname{Lip}\left(\boldsymbol{\eta}_{Q} \circ f\right)
$$

and defining $G=\mathbf{p}^{*}+\mathbf{q}^{*} \circ g, K=\mathbf{p}[Z]$ and $T \in \mathscr{D}^{\prime}\left(\mathbf{R}^{m}, \mathbf{R}^{n-m}\right)$ by

$$
T(\theta)=\int\left\langle D \theta(x), D \Psi_{0}^{\S}(D g(x))\right\rangle \mathrm{d} \mathscr{L}^{m} x \quad \text { for } \theta \in \mathscr{D}\left(\mathbf{R}^{m}, \mathbf{R}^{n-m}\right),
$$

the properties (11), (2) and (4) are evident noting 3.1 (4).

Next, it will be shown

$$
B_{w, t} \subset \mathbf{U}(a, s) \cap(\operatorname{spt}\|V\|) \sim A \subset C_{i} \cup D_{i}(w)
$$

whenever $w \in W, 0<t \leq u(w)$. The first inclusion is readily verified noting $\left|S_{\natural}-R(a)\right| \leq \varepsilon / 3$. If $z \in \mathbf{U}(a, s) \cap(\operatorname{spt}\|V\|) \sim A$, then

either $\|\delta V\| \mathbf{B}(z, t)>(2 \varepsilon / 3)\|V\|(\mathbf{B}(z, t))^{1-1 / m} \quad$ for some $0<t<2 s$,

or $\int_{\mathbf{B}(z, t)}|R(\xi)-R(a)| \mathrm{d}\|V\| \xi>(2 \varepsilon / 3)\|V\| \mathbf{B}(z, t)$ for some $0<t<2 s$.

In the first case, this implies $z \in C_{i}$, in the second case,

$$
\begin{gathered}
(2 \varepsilon / 3)\|V\| \mathbf{B}(z, t)<\int_{\mathbf{B}(z, t)}|R(\xi)-R(a)| \mathrm{d}\|V\| \xi \\
\leq \int_{\mathbf{B}(z, t)}|R(\xi)-R(w)| \mathrm{d}\|V\| \xi+|R(a)-R(w)|\|V\| \mathbf{B}(z, t), \\
(\varepsilon / 3)\|V\| \mathbf{B}(z, t)<\int_{\mathbf{B}(z, t)}|R(\xi)-R(w)| \mathrm{d}\|V\| \xi \\
\leq\|V\|(\mathbf{B}(z, t))^{1-1 / q}\left(\int_{\mathbf{B}(z, t)}|R(\xi)-R(w)|^{q} \mathrm{~d}\|V\| \xi\right)^{1 / q},
\end{gathered}
$$

hence $z \in D_{i}(w)$, and the second inclusion and hence the claim are proven. The inclusions imply the density estimate

$$
\mathbf{\Theta}^{m+\alpha q}\left(\|V\|\llcorner B, w)=\boldsymbol{\Theta}^{m+\alpha q}(\|V\|\llcorner(U \sim A), w)=0 \quad \text { whenever } w \in W .\right.
$$

Noting $a \in W$ and $\Theta^{m}(\|V\|\llcorner U \sim(W \cap J), a)=0$, one infers in particular

$$
\mathbf{\Theta}^{m}\left(\|V\|\llcorner U \sim Z, a)=0, \quad \Theta^{m}(\|V\|\llcorner Z, a)=Q\right.
$$

and it remains to verify that $g, G, K$, and $T$ satisfy (3), (5) and (6).

In preparation to this, the following tilt estimate will be shown with $\Delta_{1}=$ $\left(1+L^{2}\right)^{1 / 2}\left(1-L^{2}\right)^{-1 / 2} m^{1 / 2}$

$$
\begin{gathered}
Q^{-1 / 2}\left(\int_{\mathbf{B}(\mathbf{p}(z), t) \cap \operatorname{dmn} f}|\operatorname{ap} A f(x)(+)(-\tau)|^{r} \mathrm{~d} \mathscr{L}^{m} x\right)^{1 / r} \\
\quad \leq \Delta_{1}\left(\int_{\mathbf{C}\left(S, z, t, \delta_{4} t\right)}\left|R(\xi)-\tau_{\sharp}\right|^{r} \mathrm{~d}\|V\| \xi\right)^{1 / r}
\end{gathered}
$$

whenever $1 \leq r<\infty, z \in Z, 0<t \leq u(z), \tau \in \operatorname{Hom}\left(\mathbf{R}^{m}, \mathbf{R}^{n-m}\right)$ with $\|\tau\| \leq L$ (here the identification $\tau \subset \mathbf{R}^{m} \times \mathbf{R}^{n-m} \simeq \mathbf{R}^{n}$ is used); in fact, recalling $L \leq 1 / 8$ and $z \in \operatorname{graph}_{Q} f$, one notes

$$
\operatorname{graph}_{Q} f \mid \mathbf{B}(\mathbf{p}(z), t) \subset \mathbf{C}\left(S, z, t, \delta_{4} t\right) \subset \mathbf{C}(S, a, s, s),
$$


hence for $0<\gamma<\infty$

$$
\mathbf{B}(\mathbf{p}(z), t)) \cap\left\{x: Q^{-1 / 2} \mid \text { ap } A f(x)(+)(-\tau) \mid>\gamma\right\}
$$

is $\mathscr{H}^{m}$ almost contained in

$$
\mathbf{p}\left[\mathbf{C}\left(S, z, t, \delta_{4} t\right) \cap\left\{\xi: \Delta_{1}\left|R(\xi)-\tau_{\natural}\right|>\gamma\right\}\right]
$$

by 3.1 (4) (5) and Allard All72, 8.9(5)]. For $x \in K$, taking $z=G(x)$ and $\tau$ associated to im $R(z)$, one infers, noting $\boldsymbol{\Theta}^{m+\alpha q}\left(\mathscr{L}^{m}\left\llcorner\mathbf{R}^{m} \sim \operatorname{dmn} f, x\right)=0\right.$ by the density estimate for $B$ and $3.1(6)$ and $\Delta_{1} \leq 2 m^{1 / 2}$,

$$
\begin{aligned}
& \limsup _{t \rightarrow 0+} t^{-\beta-m / r}\left(\int_{\mathbf{B}(x, t)}|D g(\zeta)-\tau|^{r} \mathrm{~d} \mathscr{L}^{m} \zeta\right)^{1 / r} \\
& \quad \leq 2 m^{1 / 2} \limsup _{t \rightarrow 0+} t^{-\beta-m / r}\left(\int_{\mathbf{B}(z, t)}|R(\xi)-R(z)|^{r} \mathrm{~d}\|V\| \xi\right)^{1 / r}
\end{aligned}
$$

whenever $x \in K, 0<\beta \leq 1,1 \leq r<\infty$, and $\beta r \leq \alpha q$, hence in particular, taking $\beta=\alpha \inf \{1, q / r\}$ and noting that the right hand side in this case is finite by [Fed69, 2.4.17] as $z \in P$,

$$
\lim _{t \rightarrow 0+}\left(f_{\mathbf{B}(x, t)}|D g(\zeta)-\tau|^{r} \mathrm{~d} \mathscr{L}^{m} \zeta\right)^{1 / r}=0 \quad \text { for } 1 \leq r<\infty
$$

and $g$ is differentiable at $x$ with $D g(x)=\tau$ by the argument in EG92, Theorem 6.2.1]. Since $Z \subset \operatorname{im} G, K$ is $\mathscr{L}^{m}$ measurable, hence (3) and (5) are now proven and it remains to prove (6).

Choose $\eta \in \mathscr{D}^{0}\left(\mathbf{R}^{n-m}\right)$ such that

$$
\begin{gathered}
0 \leq \eta(y) \leq 1 \quad \text { for } y \in \mathbf{R}^{n-m}, \\
\operatorname{spt} \eta \subset \mathbf{U}(0,1 / 4), \quad \mathbf{B}(0,1 / 8) \subset \operatorname{Int}\left(\mathbf{R}^{n-m} \cap\{y: \eta(y)=1\}\right)
\end{gathered}
$$

and define $T_{x}$ for $x \in K$ as in (6). Fix $x \in K$, let $z=G(x)$, note $\mathbf{p}(z)=x$ and abbreviate

$$
\theta_{t}=t^{-m} \theta \circ \boldsymbol{\mu}_{1 / t} \circ \boldsymbol{\tau}_{-\mathbf{p}(z)}, \quad \eta_{t}=\eta \circ \boldsymbol{\mu}_{1 / t} \circ \boldsymbol{\tau}_{-\mathbf{q}(z)}
$$

whenever $0<t \leq u(z)$ and $\theta \in \mathscr{D}\left(\mathbf{R}^{m}, \mathbf{R}^{n-m}\right)$. The remaining estimate will carried out by showing that

$$
\begin{gathered}
Q T_{x}\left(\theta_{t}\right)-(\delta V)\left(\left(\eta_{t} \circ \mathbf{q}\right) \cdot\left(\mathbf{q}^{*} \circ \theta_{t} \circ \mathbf{p}\right)\right), \\
(\delta V)\left(\left(\eta_{t} \circ \mathbf{q}\right) \cdot\left(\mathbf{q}^{*} \circ \theta_{t} \circ \mathbf{p}\right)\right)-Q \int\left\langle D \theta_{t}(\zeta), D \Psi_{0}^{\S}(D g(\zeta))\right\rangle \mathrm{d} \mathscr{L}^{m} \zeta
\end{gathered}
$$

both tend to 0 as $t \rightarrow 0+$ uniformly with respect to $\theta \in \mathscr{D}\left(\mathbf{R}^{m}, \mathbf{R}^{n-m}\right)$ such that $\operatorname{spt} \theta \subset \mathbf{U}(0,1)$ and $|D \theta|_{\infty ; 0,1} \leq 1$.

To prove the first estimate, one notes that the conditions $\Theta^{m-1}(\|\delta V\|, z)=$ $0, \Theta^{m}(\|V\|, z)=Q$ and $z \in P$ imply, for example using Allard [All72, 6.4,5] and [Men10, 3.1],

$$
t^{-m} \int \phi\left(t^{-1}(\xi-z), \operatorname{im} R(\xi)\right) \mathrm{d}\|V\| \xi \rightarrow Q \int_{\operatorname{im} R(z)} \phi(\xi, \operatorname{im} R(z)) \mathrm{d} \mathscr{H}^{m} \xi
$$

as $t \rightarrow 0+$ whenever $\phi \in \mathscr{K}\left(\mathbf{R}^{n} \times \mathbf{G}(n, m)\right)$. Since also, noting

$$
\begin{gathered}
\left(\eta_{t} \circ \mathbf{q}\right) \cdot\left(\mathbf{q}^{*} \circ \theta_{t} \circ \mathbf{p}\right)=t^{-m}\left((\eta \circ \mathbf{q}) \cdot\left(\mathbf{q}^{*} \circ \theta \circ \mathbf{p}\right)\right) \circ \boldsymbol{\mu}_{1 / t} \circ \boldsymbol{\tau}_{-z}, \\
\mathbf{C}(T, 0,1) \cap \operatorname{Tan}^{m}(\|V\|, z) \subset \mathbf{C}(T, 0,1,1 / 8)
\end{gathered}
$$


as $L \leq 1 / 8$ and $z \in \operatorname{graph}_{Q} f$, one readily uses the conditions on $\delta V$ and $\mathbf{h}(V ; \cdot)$ imposed by the fact $z \notin N$ to infer

$$
\begin{aligned}
\lim _{t \rightarrow 0+} & (\delta V)\left(\left(\eta_{t} \circ \mathbf{q}\right) \cdot\left(\mathbf{q}^{*} \circ \theta_{t} \circ \mathbf{p}\right)\right) \\
\quad & =-Q \int_{\operatorname{im} R(z)} \mathbf{h}(V ; z) \bullet(\eta \circ \mathbf{q})(\xi)\left(\mathbf{q}^{*} \circ \theta \circ \mathbf{p}\right)(\xi) \mathrm{d} \mathscr{H}^{m} \xi \\
& =-Q \int \Psi_{0}^{\S}(D g(x)) \mathbf{h}(V ; z) \bullet\left(\mathbf{q}^{*} \circ \theta\right)(\zeta) \mathrm{d} \mathscr{L}^{m} \zeta=Q T_{x}\left(\theta_{t}\right)
\end{aligned}
$$

and the convergence is uniform with respect to $\theta \in \mathscr{D}\left(\mathbf{R}^{m}, \mathbf{R}^{n-m}\right)$ such that $\operatorname{spt} \theta \subset \mathbf{U}(0,1)$ and $|D \theta|_{\infty: 0.1} \leq 1$ as this family of functions is compact with respect to $|\cdot|_{\infty ; 0,1}$ by $\left[\right.$ Fed69, 2.10.21] and $\Theta^{* m}(\|\delta V\|, z)<\infty$.

To prove the second estimate, define

$$
\gamma_{1}=\sup \left\|D^{2} \Psi_{0}^{\S}\right\|\left[\mathbf{B}\left(0, m^{1 / 2} L\right)\right], \quad \gamma_{2}=\operatorname{Lip}\left(D^{2} \Psi_{0}^{\S} \mid \mathbf{B}\left(0,3 m^{1 / 2} L\right)\right) .
$$

Apply 3.1(7) with $\tau=D g(x)$ and $0<t \leq u(z)$ to obtain

$$
\begin{aligned}
&\left|Q \int\left\langle D \theta_{t}(x), D \Psi_{0}^{\S}(D g(x))\right\rangle \mathrm{d} \mathscr{L}^{m} x-(\delta V)\left(\left(\eta_{t} \circ \mathbf{q}\right) \cdot\left(\mathbf{q}^{*} \circ \theta_{t} \circ \mathbf{p}\right)\right)\right| \\
& \leq \gamma_{1} Q m^{1 / 2} L \int_{C_{z, t}}\left|D \theta_{t}\right| \mathrm{d} \mathscr{L}^{m} \\
& \quad+\gamma_{2} \int_{E_{z, t} \sim C_{z, t}}\left|D \theta_{t}(\zeta)\right||\operatorname{ap} A f(\zeta)(+)(-D g(x))|^{2} \mathrm{~d} \mathscr{L}^{m} \zeta \\
& \quad+m^{1 / 2} \int_{D_{z, t}}\left|D\left(\left(\eta_{t} \circ \mathbf{q}\right) \cdot\left(\mathbf{q}^{*} \circ \theta_{t} \circ \mathbf{p}\right)\right)\right| \mathrm{d}\|V\| .
\end{aligned}
$$

The first and the third summand on the right hand side may be estimated by use of 3.1 (6) as follows

$$
\begin{gathered}
\int_{C_{z, t}}\left|D \theta_{t}\right| \mathrm{d} \mathscr{L}^{m} \leq t^{-m-1} \mathscr{L}^{m}\left(C_{z, t}\right) \leq \Delta_{2} t^{-m-1}\|V\|\left(B_{z, t}\right), \\
\int_{D_{z, t}}\left|D\left(\left(\eta_{t} \circ \mathbf{q}\right) \cdot\left(\mathbf{q}^{*} \circ \theta_{t} \circ \mathbf{p}\right)\right)\right| \mathrm{d}\|V\| \\
\leq t^{-m-1}\left(1+|D \eta|_{\infty ; 0,1}\right)\|V\|\left(D_{z, t}\right) \leq \Delta_{2} t^{-m-1}\left(1+|D \eta|_{\infty ; 0,1}\right)\|V\|\left(B_{z, t}\right)
\end{gathered}
$$

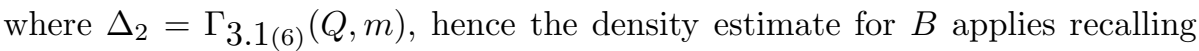
$\alpha q \geq 1$. To estimate the remaining summand, one computes

$$
\begin{aligned}
\int_{E_{z, t}} \sim C_{z, t}\left|D \theta_{t}(\zeta)\right||\operatorname{ap} A f(\zeta)(+)(-D g(x))|^{2} \mathrm{~d} \mathscr{L}^{m} \zeta \\
\quad \leq t^{-1-m} \int_{\mathbf{B}(x, t) \cap \operatorname{dmn} f}|\operatorname{ap} A f(\zeta)(+)(-D g(x))|^{2} \mathrm{~d} \mathscr{L}^{m} \zeta,
\end{aligned}
$$

uses the tilt estimate and recalls $z \in P^{\prime}$.

3.4 Remark. It would significantly simplify the treatment in 2.132 .17 if one could obtain an estimate in $|\cdot|_{-1, r ; a, s}$ in (6) for some $r>1$. However, in this case it seems to be unclear how to control the integral over $D_{z, t}$ in the last paragraph as this set may contain arbitrarily steep parts of the varifold, see Brakke's example in Bra78, 6.1].

3.5. If $f: \mathbf{R}^{m} \rightarrow \mathbf{R}^{n-m}$ is a linear map, $v \in \mathbf{R}^{n}$ is orthogonal to $\operatorname{im}\left(\mathbf{p}^{*}+\mathbf{q}^{*} \circ f\right)$ then $v \in \operatorname{ker}\left(\mathbf{p}^{*}+\mathbf{q}^{*} \circ f\right)^{*}, \mathbf{p}(v)=-\left(f^{*} \circ \mathbf{q}\right)(v)$ and

$$
\left(\mathbf{q}^{*}-\mathbf{p}^{*} \circ f^{*}\right)(\mathbf{q}(v))=v .
$$


3.6 Theorem. Suppose $m, n \in \mathscr{P}, m<n, U$ is an open subset of $\mathbf{R}^{n}, V \in$ $\mathbf{I V}_{m}(U)$ and $\|\delta V\|$ is a Radon measure.

Then there exists a countable collection $C$ of $m$ dimensional submanifolds of $\mathbf{R}^{n}$ of class 2 such that $\|V\|(U \sim \bigcup C)=0$ and each member $M$ of $C$ satisfies

$$
\mathbf{h}(V ; z)=\mathbf{h}(M ; z) \quad \text { for }\|V\| \text { almost all } z \in U \cap M .
$$

Proof. First, note that for $\|V\|$ almost all $z \in U$ there holds $\operatorname{Tan}^{m}(\|V\|, z) \in$ $\mathbf{G}(n, m)$ and

$$
\lim _{r \rightarrow 0+} r^{-1 / 2-m / 2}\left(\int_{\mathbf{B}(z, r) \times \mathbf{G}(n, m)}\left|S_{\natural}-\operatorname{Tan}^{m}(\|V\|, z)_{\natural}\right|^{2} \mathrm{~d} V(\xi, S)\right)^{1 / 2}=0
$$

by Brakke Bra78, 5.7,5] or [Men09b, 8.6]. Let $\Psi$ denote the area integrand, abbreviate $\Phi=\Psi_{0}^{\S}$ and note $D^{2} \Phi(0)=\Upsilon$ with $\Upsilon$ as in 2.1 by [Fed69, 5.1.9]. Define $\varepsilon=q_{2.17}(m, n), \Delta=\Gamma_{[2.20}(m(n-m), 2), s=\varepsilon / \Delta$ and choose $0<\delta<$ $\infty$ such that

$$
\left\|D^{2} \Phi(\sigma)-D^{2} \Phi(0)\right\| \leq s \quad \text { whenever } \sigma \in \operatorname{Hom}\left(\mathbf{R}^{m}, \mathbf{R}^{n-m}\right) \cap \mathbf{B}(0, \delta) .
$$

Applying 2.20 with $H, k, l, a$ replaced by $\operatorname{Hom}\left(\mathbf{R}^{m}, \mathbf{R}^{n-m}\right), 2,3$, 0, one obtains $F: \operatorname{Hom}\left(\mathbf{R}^{m}, \mathbf{R}^{n-m}\right) \rightarrow \mathbf{R}$ of class 3 such that

$$
\begin{gathered}
D^{i} F(\sigma)=D^{i} \Phi(\sigma) \text { for } i=\{0,1,2\}, \sigma \in \operatorname{Hom}\left(\mathbf{R}^{m}, \mathbf{R}^{n-m}\right) \cap \mathbf{B}(0, \delta / 2), \\
\left\|D^{2} F(\sigma)-D^{2} \Phi(0)\right\| \leq \Delta s=\varepsilon \quad \text { whenever } \sigma \in \operatorname{Hom}\left(\mathbf{R}^{m}, \mathbf{R}^{n-m}\right), \\
D^{3} F \text { has compact support, }
\end{gathered}
$$

hence $\operatorname{Lip} D^{2} F<\infty$. Define $L=m^{-1 / 2} \delta / 2$ and apply 3.3 with $p, q, \alpha$ replaced by $1,2,1 / 2$ to obtain $P$ and $H$ with the properties listed there. Fix $Z \in H$ and take $\pi_{1} \in O$ and $\pi_{2}, g, G, K$ as in 3.3 to infer from 2.17, 2.18 and 3.3 (6), noting 3.3 (5) with $\beta=1 / 2$ and $r=2$, the existence a sequence of functions $u_{i}: \mathbf{R}^{m} \rightarrow \mathbf{R}^{n-m}$ of class 2 such that with $A_{i}=K \cap\left\{x: g(x)=u_{i}(x)\right\}$ for $i \in \mathscr{P}$

$$
\left\langle D^{2} u_{i}(x), C_{F}\left(D u_{i}(x)\right)\right\rangle=\Phi\left(D u_{i}(x)\right) \pi_{2}(\mathbf{h}(V ; G(x)))
$$

for $\mathscr{L}^{m}$ almost all $x \in A_{i}$. Defining $M_{i}=\operatorname{im}\left(\pi_{1}^{*}+\pi_{2}^{*} \circ u_{i}\right)$ and noting

$$
\left\langle D^{2} u_{i}(x), C_{\Phi}\left(D u_{i}(x)\right)\right\rangle=\Phi\left(D u_{i}(x)\right) \pi_{2}\left(\mathbf{h}\left(M_{i} ;\left(\pi_{1}^{*}+\pi_{2}^{*} \circ u_{i}\right)(x)\right)\right)
$$

for $x \in \mathbf{R}^{m}$ where $C_{\Phi}$ is as in 2.1 and

$$
\begin{gathered}
C_{\Phi}(\sigma)=C_{F}(\sigma) \quad \text { for } \sigma \in \operatorname{Hom}\left(\mathbf{R}^{m}, \mathbf{R}^{n-m}\right) \cap \mathbf{B}(0, \delta / 2), \\
\left|D u_{i}(x)\right|=|D g(x)| \leq L m^{1 / 2}=\delta / 2 \text { for } \mathscr{L}^{m} \text { almost all } x \in A_{i}
\end{gathered}
$$

by 3.3 (2), one concludes

$$
\pi_{2}(\mathbf{h}(V ; G(x)))=\pi_{2}\left(\mathbf{h}\left(M_{i} ; G(x)\right)\right) \quad \text { for } \mathscr{L}^{m} \text { almost all } x \in A_{i},
$$

hence by 3.5 since $\mathbf{h}(V ; z) \in \operatorname{Nor}^{m}(\|V\|, z)$ for $\|V\|$ almost all $z$ by Brakke Bra78, 5.8],

$$
\mathbf{h}(V ; G(x))=\mathbf{h}\left(M_{i} ; G(x)\right) \quad \text { for } \mathscr{L}^{m} \text { almost all } x \in A_{i} .
$$

Finally, recall $\|V\|(U \sim P)=0$. 
3.7 Remark. One could also prove Brakke Bra78, 5.8] instead of using it. Since the proof then still yields a collection $C$ with all properties except of the last one, one can define a $\|V\|$ measurable function $h$ such that for $\|V\|$ almost all $z \in U$ there holds $h(z)=\mathbf{h}(M ; z)$ whenever $z \in U \cap M$ and $M \in C$. Following the above proof, one obtains

$$
\pi_{2}(\mathbf{h}(V ; G(x)))=\pi_{2}(h(G(x))) \quad \text { for } \mathscr{L}^{m} \text { almost all } x \in A_{i}
$$

whenever $\pi_{1} \in O, \pi_{2} \in \mathbf{O}^{*}(n, n-m)$ with $\pi_{2} \circ \pi_{1}^{*}=0$, and, as $O$ is open, this suffices to conclude

$$
\mathbf{h}(V ; G(x))=h(G(x)) \in \operatorname{Nor}^{m}(\|V\|, G(x)) \quad \text { for } \mathscr{L}^{m} \text { almost all } x \in A_{i} .
$$

3.8 Remark. Noting Fed69, 2.10.19(4)], one infers that the function mapping $\|V\|$ almost all $z$ onto $\operatorname{Tan}^{m}(\|V\|, z)_{\natural} \in \operatorname{Hom}\left(\mathbf{R}^{n}, \mathbf{R}^{n}\right)$ is $(\|V\|, m)$ approximately differentiable at $\|V\|$ almost all $z$.

Therefore, combining 3.6 with Mantegazza Man96, Remark 3.9, Theorem 5.4 , one obtains the following proposition on curvature varifolds with boundary in the sense of Mantegazza [Man96, Definition 3.1]: If $V$ is a curvature varifold with boundary in an open subset $U$ of $\mathbf{R}^{n}$ then then there exists a countable collection $C$ of $m$ dimensional submanifolds of $\mathbf{R}^{n}$ of class 2 such that $\|V\|(U \sim \cup C)=0$ and such that for each member $M$ of $C$ the second fundamenal forms of $V$ and $M$ agree at $\|V\|$ almost every $z \in U \cap M$. Clearly, this includes curvature varifolds in the sense of Hutchinson Hut86, 5.2.3].

\section{Applications to decay rates of tilt-excess for integral varifolds}

The present section discusses some consequences of 3.6 in terms of decay and differentiability of tilt quantities.

4.1 Lemma. Suppose $m, n, Q \in \mathscr{P}, m<n$, either $p=m=1$ or $1<p<m=2$ or $1 \leq p<m>2$ and $\frac{m p}{m-p}=2,0<\delta \leq 1$, and $1 \leq M<\infty$.

Then there exist positive, finite numbers $\varepsilon$ and $\Gamma$ with the following property.

If $a \in \mathbf{R}^{n}, 0<r<\infty, V \in \mathbf{I V}_{m}(\mathbf{U}(a, 6 r)), \psi$ and $p$ are related to $V$ as in 3.2. $T \in \mathbf{G}(n, m), Z$ is a $\|V\|$ measurable subset of $\mathbf{C}(T, a, r, 3 r)$,

$$
\begin{gathered}
(Q-1 / 2) \boldsymbol{\alpha}(m) r^{m} \leq\|V\|(\mathbf{C}(T, a, r, 3 r)) \leq(Q+1 / 2) \boldsymbol{\alpha}(m) r^{m}, \\
\|V\|(\mathbf{C}(T, a, r, 4 r) \sim \mathbf{C}(T, a, r, r)) \leq(1 / 2) \boldsymbol{\alpha}(m) r^{m}, \\
\|V\| \mathbf{U}(a, 6 r) \leq M \boldsymbol{\alpha}(m) r^{m}, \quad\|V\|(\mathbf{C}(T, a, r / 2, r / 2)) \geq(Q-1 / 4) \boldsymbol{\alpha}(m)(r / 2)^{m}, \\
\|V\|(\mathbf{C}(T, a, r, 3 r) \sim Z) \leq \varepsilon \boldsymbol{\alpha}(m) r^{m}, \quad\left(\int\left|S_{\natural}-T_{\natural}\right|^{2} \mathrm{~d} V(z, S)\right)^{1 / 2} \leq \varepsilon r^{m / 2},
\end{gathered}
$$

then

$$
\begin{aligned}
& \left(r^{-m} \int_{\mathbf{C}(T, a, r / 4, r / 4) \times \mathbf{G}(n, m)}\left|S_{\natural}-T_{\natural}\right|^{2} \mathrm{~d} V(z, S)\right)^{1 / 2} \\
& \leq \quad \delta\left(r^{-m} \int_{\mathbf{C}(T, a, r, r) \times \mathbf{G}(n, m)}\left|S_{\natural}-T_{\natural}\right|^{2} \mathrm{~d} V(z, S)\right)^{1 / 2} \\
& \quad+\Gamma\left(r^{-m-1} \int_{Z} \operatorname{dist}(z-a, T) \mathrm{d}\|V\| z+r^{1-m / p} \psi(\mathbf{U}(a, 6 r))^{1 / p}\right) .
\end{aligned}
$$

Proof. See [Men09b, 7.5]. 
4.2 Theorem. Suppose $m, n, p, U$, and $V$ are as in 3.2, $V \in \mathbf{I V}_{m}(U)$ and

$$
\phi(a, r, T)=\left(r^{-m} \int_{\mathbf{U}(a, r) \times \mathbf{G}(n, m)}\left|S_{\natural}-T_{\natural}\right|^{2} \mathrm{~d} V(z, S)\right)^{1 / 2}
$$

whenever $a \in \mathbf{R}^{n}, 0<r<\infty, \mathbf{U}(a, r) \subset U$, and $T \in \mathbf{G}(n, m)$.

Then the following two statements hold:

(1) If either $m=2$ and $0<\tau<1$ or $\sup \{2, p\}<m$ and $\tau=\frac{m p}{2(m-p)}<1$ then

$$
\lim _{r \rightarrow 0+} r^{-\tau} \phi(a, r, T)=0 \quad \text { for } V \text { almost all }(a, T) \in U \times \mathbf{G}(n, m) .
$$

(2) If either $m=1$ or $m=2$ and $p>1$ or $m>2$ and $p \geq 2 m /(m+2)$ then

$$
\limsup _{r \rightarrow 0+} r^{-1} \phi(a, r, T)<\infty \quad \text { for } V \text { almost all }(a, T) \in U \times \mathbf{G}(n, m) .
$$

Proof of (11). From 3.6 one obtains a sequence of maps $R_{i}: U \rightarrow \operatorname{Hom}\left(\mathbf{R}^{n}, \mathbf{R}^{n}\right)$ of class 1 such that the sets $A_{i}=U \cap\left\{z: R_{i}(z)=\operatorname{Tan}^{m}(\|V\|, z)_{\natural}\right\}$ cover $\|V\|$ almost all of $U$. By [Men09b, 8.6] and [Men09a, 3.7 (i)] one infers

$$
\lim _{r \rightarrow 0+} r^{-\tau-m / 2}\left(\int_{\mathbf{B}(z, r) \times \mathbf{G}(n, m)}\left|R_{i}(z)-S_{\natural}\right|^{2} \mathrm{~d} V(\xi, S)\right)^{1 / 2}=0
$$

for $\|V\|$ almost all $z \in A_{i}$ and the conclusion follows.

Proof of (2). Assume that either $p=m=1$ or $1<p<m=2$ or $1 \leq p<m>$ 2 and $\frac{m p}{m-p}=2$. Choose $C$ as in 3.6. Then by 3.6] and [Fed69, 2.10.19(4), 2.9.5] for $\|V\|$ almost all $a \in U$ there holds for some $Q \in \mathscr{P}, T \in \mathbf{G}(n, m)$ and some $M \in C$

$$
\begin{gathered}
T=\operatorname{Tan}(M, a), \quad \boldsymbol{\Theta}^{m}(\|V\|\llcorner U \sim M, a)=0, \\
\limsup _{r \rightarrow 0+} r^{-m / p} \psi(\mathbf{B}(a, r))^{1 / p}<\infty, \\
r^{-m} \int \phi\left(r^{-1}(z-a), S\right) \mathrm{d} V(z, S) \rightarrow Q \int_{T} \phi(z, T) \mathrm{d} \mathscr{H}^{m} z \quad \text { as } r \rightarrow 0+
\end{gathered}
$$

whenever $\phi \in \mathscr{K}\left(\mathbf{R}^{n} \times \mathbf{G}(n, m)\right)$. Note that

$$
\limsup _{r \rightarrow 0+} r^{-m-2} \int_{\mathbf{C}(T, a, r, 3 r) \cap M} \operatorname{dist}(z-a, T) \mathrm{d}\|V\| z<\infty
$$

as $M$ is submanifold of class 2 . It follows with $\delta=2^{-m-3}, \Delta_{1}=7^{m} Q$ that there exist $0<R<\infty$ and $0 \leq \gamma<\infty$ such that $\mathbf{U}(a, 6 R) \subset U$,

$$
r^{-m-1} \int_{\mathbf{C}(T, a, r, 3 r) \cap M} \operatorname{dist}(z-a, T) \mathrm{d}\|V\| z+r^{1-m / p} \psi(\mathbf{U}(a, 6 r))^{1 / p} \leq \gamma r
$$

for $0<r \leq R$, and $V$ satisfies the hypotheses of 4.1 for each $0<r \leq R$ with $\varepsilon=\varepsilon 4.1\left(m, n, Q, p, \delta, \Delta_{1}\right)$ and $M, Z$ replaced by $\Delta_{1}, \mathbf{C}(T, a, r, 3 r) \cap M$. With $f(r)=r^{-m / 2}\left(\int_{\mathbf{C}(T, a, r, r) \times \mathbf{G}(n, m)}\left|S_{\natural}-T_{\natural}\right|^{2} \mathrm{~d} V(z, S)\right)^{1 / 2}$ for $0<r \leq R$ one defines

$$
\Delta_{2}=\Gamma \overline{4.1}\left(m, n, Q, p, \delta, \Delta_{1}\right), \quad \Delta_{3}=\sup \left\{2^{m+3} \Delta_{2} \gamma, 2^{m+2} R^{-1} f(R)\right\},
$$


one inductively infers from 4.1

$$
f(r) \leq \Delta_{3} r \quad \text { whenever } 0<r \leq R
$$

in fact it holds for $R / 4 \leq r \leq R$ and, provided it holds for $r$,

$$
f(r / 4) \leq 2^{m}\left(\delta \Delta_{3} r+\Delta_{2} \gamma r\right) \leq \Delta_{3}(r / 4)
$$

by 4.1. The conclusion is now evident.

4.3 Remark. Having 3.6 at one's disposal, the proof of (2) follows Schätzle in Sch09, Theorem 3.1] where the case $p \geq 2$ is treated. In extending the result to the present case, the main difference is the use of the coercive estimate in Men09b, 3.9] in the proof of 4.1 replacing the use of corresponding estimate in Brakke [Bra78, 5.5] (see also Allard [All72, 8.13]).

4.4 Remark. For both parts the family of examples provided in [Men09a, 1.2] shows that if $m>2$ then $p$ cannot be replaced by any smaller number, see Men09b, 8.7].

4.5 Remark. In case of (2) combining this result with [Men09a, 3.9], one obtains

$$
f_{\mathbf{B}(a, r)}(\mid R(z)-R(a)-\langle R(a)(z-a), \text { ap } D R(a)\rangle|/| z-a \mid)^{2} \mathrm{~d}\|V\| z \rightarrow 0
$$

as $r \rightarrow 0+$ for $\|V\|$ almost all $a$ where $R(z)=\operatorname{Tan}^{m}(\|V\|, z)_{\natural}$ and the approximate differential is taken with respect to $(\|V\|, m)$.

4.6 Remark. Clearly, one can also obtain decay results for height quantities from this result by use of [Men10, 4.11].

\section{A Lebesgue points for a distribution}

In this Appendix the part $q=1$ of Theorem 4 of the Introduction is provided. Its purpose is to clarify the relations of the sets $A_{1}$ and $A_{2}$ occurring in 2.17 .

A.1 Lemma. Suppose $m, n \in \mathscr{P}, m<n, A$ is a closed subset of $\mathbf{R}^{m}, R \in$ $\mathscr{D}^{\prime}\left(\mathbf{R}^{m}, \mathbf{R}^{n-m}\right)$, dist( $\left.\operatorname{spt} R, A\right)>0,0 \leq \gamma<\infty$, and $0<r<\infty$ such that

$$
|R|_{-1,1 ; x, \varrho} \leq \gamma \varrho^{m+1} \quad \text { whenever } 0<\varrho<5 r, x \in A .
$$

Then

$$
|R|_{-1,1 ; a, r} \leq \Gamma \gamma r \mathscr{L}^{m}(\mathbf{B}(a, 4 r) \sim A) \quad \text { for } a \in A
$$

where $\Gamma$ is a positive, finite number depending only on $m$.

Proof. Assume $r \leq \frac{2}{9}$, let $a \in A, \theta \in \mathscr{D}\left(\mathbf{R}^{m}, \mathbf{R}^{n-m}\right)$ with $\operatorname{spt} \theta \subset \mathbf{U}(a, r)$, choose $0<\varepsilon \leq \inf \{r, \operatorname{dist}(\operatorname{spt} R, A)\}$, define

$$
B=\mathbf{R}^{m} \cap\{x: \operatorname{dist}(x, \operatorname{spt}(R\llcorner\theta)) \leq \varepsilon / 2\}
$$

where $R\left\llcorner\theta \in \mathscr{E}_{0}\left(\mathbf{R}^{m}\right)\right.$ is defined by $\left(R\llcorner\theta)(v)=R(v \theta)\right.$ for $v \in \mathscr{E}^{0}\left(\mathbf{R}^{m}\right)$, and apply [Fed69, 3.1.13] to obtain $S, v_{s}$, and $h$ with $\Phi=\left\{\mathbf{R}^{m} \sim A, \mathbf{R}^{m} \sim B\right\}$; in particular $S$ is a countable subset of $\bigcup \Phi$,

$$
h(x)=\frac{1}{20} \sup \{\inf \{1, \operatorname{dist}(x, A)\}, \inf \{1, \operatorname{dist}(x, B)\}\} \quad \text { for } x \in \bigcup \Phi
$$


and $v_{s}$ for $s \in S$ form a partition of unity on $\bigcup \Phi$ with $\operatorname{spt} v_{s} \subset \mathbf{B}(s, 10 h(s))$ for $s \in S$. Noting $\bigcup \Phi=\mathbf{R}^{m}$, one defines $T=S \cap\left\{s: B \cap \operatorname{spt} v_{s} \neq \emptyset\right\}$ and infers

$$
\sum_{s \in S \sim T} v_{s}(x)=0 \quad \text { for } x \in \mathbf{R}^{m} \text { with } \operatorname{dist}(x, \operatorname{spt}(R\llcorner\theta))<\varepsilon / 2,
$$

hence $\left(R\llcorner\theta)\left(\sum_{s \in S \sim T} v_{s}\right)=0\right.$ and

$$
R(\theta)=R\left(\left(\sum_{s \in T} v_{s}\right) \theta\right)=\sum_{s \in T} R\left(v_{s} \theta\right) .
$$

Choose $\xi(s) \in A$ for each $s \in T$ such that $|s-\xi(s)|=\operatorname{dist}(s, A)$. If $s \in T$ then there exists $y \in B \cap \operatorname{spt} v_{s} \subset \mathbf{B}(a, r+\varepsilon / 2)$ and one observes

$$
\begin{gathered}
\operatorname{dist}(y, A) \leq|y-a| \leq r+\varepsilon / 2 \leq(3 / 2) r \leq \frac{1}{3}<1, \quad h(y)=\frac{1}{20} \operatorname{dist}(y, A), \\
|s-y| \leq 10 h(s) \leq 10 h(y)+\frac{1}{2}|s-y|, \quad|s-y| \leq 20 h(y)=\operatorname{dist}(y, A) \leq|y-a|, \\
\operatorname{dist}(s, A) \leq|s-y|+\operatorname{dist}(y, A) \leq 2 \operatorname{dist}(y, A) \leq 3 r \leq \frac{2}{3}<1, \\
B \cap \mathbf{B}(s, 10 h(s)) \neq \emptyset, \quad \frac{1}{20} \operatorname{dist}(s, B) \leq \frac{1}{2} h(s), \quad 0<h(s)=\frac{1}{20} \operatorname{dist}(s, A), \\
|s-\xi(s)| \leq|s-a| \leq|s-y|+|y-a| \leq 2 r+\varepsilon \leq 3 r \leq \frac{2}{3}, \\
\mathbf{B}(s, h(s)) \subset \mathbf{B}(a, 4 r) \sim A .
\end{gathered}
$$

Moreover, for any $x \in \mathbf{B}(s, 10 h(s)), s \in T$

$$
\begin{gathered}
|x-\xi(s)| \leq|x-s|+|s-\xi(s)| \leq(3 / 2)|s-\xi(s)|<5 r, \\
\operatorname{spt} v_{s} \subset \mathbf{B}(\xi(s),(3 / 2)|s-\xi(s)|), \\
\operatorname{dist}(s, A) \leq \operatorname{dist}(x, A)+|x-s| \leq \operatorname{dist}(x, A)+\frac{1}{2} \operatorname{dist}(s, A), \\
|s-\xi(s)|=\operatorname{dist}(s, A) \leq 2 \operatorname{dist}(x, A), \\
\operatorname{dist}(x, A) \leq \operatorname{dist}(s, A)+|x-s| \leq \frac{3}{2} \operatorname{dist}(s, A) \leq 1, \\
h(x) \geq \frac{1}{20} \operatorname{dist}(x, A) \geq \frac{1}{40}|s-\xi(s)| .
\end{gathered}
$$

Using the estimates of the preceding paragraph and the estimates of $\left|D v_{s}\right|$ given in [Fed69, 3.1.13], one infers for $s \in T$, since $\theta$ has compact support in $\mathbf{U}(a, r)$,

$$
\begin{gathered}
\left|\left(D v_{s}\right) \theta\right|_{\infty ; a, r} \leq 40 \Delta|s-\xi(s)|^{-1} r|D \theta|_{\infty ; a, r}, \\
\left|D\left(v_{s} \theta\right)\right|_{\infty ; a, r} \leq 40 \Delta\left(|s-\xi(s)|^{-1} r+1\right)|D \theta|_{\infty ; a, r}
\end{gathered}
$$

where $\Delta$ is a positive, finite number depending only on $m$ with $40 \Delta \geq 1$, hence

$$
\begin{aligned}
\left|R\left(v_{s} \theta\right)\right| & \leq \gamma(3 / 2)^{m+1}|s-\xi(s)|^{m+1} 40 \Delta\left(|s-\xi(s)|^{-1} r+1\right)|D \theta|_{\infty ; a, r} \\
& =\gamma(3 / 2)^{m+1} 40 \Delta|s-\xi(s)|^{m}(r+|s-\xi(s)|)|D \theta|_{\infty ; a, r} \\
& \leq \gamma 160 \Delta(3 / 2)^{m+1} \boldsymbol{\alpha}(m)^{-1}(20)^{m} r \mathscr{L}^{m}(\mathbf{B}(s, h(s)))|D \theta|_{\infty ; a, r} .
\end{aligned}
$$

Recalling from [Fed69, 3.1.13] that the family $\{\mathbf{B}(s, h(s)): s \in S\}$ is disjointed, one concludes

$$
|R(\theta)| \leq \Gamma \gamma r \mathscr{L}^{m}(\mathbf{B}(a, 4 r) \sim A)|D \theta|_{\infty ; a, r}
$$

where $\Gamma=8(30)^{m+1} \Delta \boldsymbol{\alpha}(m)^{-1}$. 
A.2 Remark. Some ideas of the proof were taken from Calderón and Zygmund [CZ61, Theorem 10] and [Fed69, 2.9.17].

A.3 Theorem. Suppose $m, n \in \mathscr{P}, m<n, U$ is an open subset of $\mathbf{R}^{m}$, $T \in \mathscr{D}^{\prime}\left(U, \mathbf{R}^{n-m}\right)$, and $A$ denotes the set of all $a \in U$ such that

$$
\limsup _{r \rightarrow 0+} r^{-1-m}|T|_{-1,1 ; a, r}<\infty .
$$

Then $A$ is a Borel set and for $\mathscr{L}^{m}$ almost all a $\in A$ there exists a unique constant distribution $T_{a} \in \mathscr{D}^{\prime}\left(U, \mathbf{R}^{n-m}\right)$ such that

$$
\lim _{r \rightarrow 0+} r^{-1-m}\left|T-T_{a}\right|_{-1,1 ; a, r}=0 .
$$

Moreover, $T_{a}$ depends $\mathscr{L}^{m}\llcorner A$ measurably on a.

Proof. The conclusion is local, hence one may assume spt $T$ to be compact and $U=\mathbf{R}^{m}$. Since $|T|_{-1,1 ; a, r}$ depends lower semicontinuously on $(a, r)$, the sets

$$
A_{i}=\mathbf{R}^{m} \cap\left\{a:|T|_{-1,1 ; a, r} \leq i r^{m+1} \text { for } 0<r<(10) / i\right\}
$$

defined for $i \in \mathscr{P}$ are closed. Observing $A=\bigcup\left\{A_{i}: i \in \mathscr{P}\right\}$, the conclusion will be shown to hold for $\mathscr{L}^{m}$ almost all $a \in A_{i}$.

Let $0<\varepsilon<5 / i$, choose $\Phi \in \mathscr{D}^{0}\left(\mathbf{R}^{m}\right)$ with $\int \Phi \mathrm{d} \mathscr{L}^{m}=1, \operatorname{spt} \Phi \subset \mathbf{U}(0,1)$ and define $\Phi_{\varepsilon}(x)=\varepsilon^{-m} \Phi\left(\varepsilon^{-1} x\right)$ for $x \in \mathbf{R}^{m}$,

$$
T_{\varepsilon}(\theta)=T\left(\Phi_{\varepsilon} * \theta\right)=\int f_{\varepsilon} \bullet \theta \mathrm{d} \mathscr{L}^{m} \quad \text { for } \theta \in \mathscr{D}\left(\mathbf{R}^{m}, \mathbf{R}^{n-m}\right)
$$

with $f_{\varepsilon} \in \mathscr{E}\left(\mathbf{R}^{m}, \mathbf{R}^{n-m}\right)$ given by

$$
z \bullet f_{\varepsilon}(x)=T_{y}\left(\Phi_{\varepsilon}(y-x) z\right) \quad \text { whenever } x \in \mathbf{R}^{m} \text { and } z \in \mathbf{R}^{n-m},
$$

see [Fed69, 4.1.2]. Clearly $T_{\varepsilon} \rightarrow T$ as $\varepsilon \rightarrow 0+$ and

$$
\left|f_{\varepsilon}(x)\right| \leq i 2^{m+1}|D \Phi|_{\infty ; 0,1} \quad \text { for } x \in \mathbf{R}^{m}, a \in A_{i} \text { with }|x-a| \leq \varepsilon .
$$

One defines $a_{\varepsilon}$ to be the characteristic function of $\mathbf{R}^{m} \cap\left\{x: \operatorname{dist}\left(x, A_{i}\right) \leq \varepsilon\right\}$ and $S_{\varepsilon}, R_{\varepsilon} \in \mathscr{D}^{\prime}\left(\mathbf{R}^{m}, \mathbf{R}^{n-m}\right)$ by

$$
S_{\varepsilon}(\theta)=\int a_{\varepsilon} f_{\varepsilon} \bullet \theta \mathrm{d} \mathscr{L}^{m} \quad \text { for } \theta \in \mathscr{D}\left(\mathbf{R}^{m}, \mathbf{R}^{n-m}\right), \quad R_{\varepsilon}=T_{\varepsilon}-S_{\varepsilon} .
$$

Estimating for $a \in A_{i}, 0<\varrho<5 r<5 / i, \theta \in \mathscr{D}\left(\mathbf{R}^{m}, \mathbf{R}^{n-m}\right)$ with $\operatorname{spt} \theta \subset$ $\mathbf{U}(a, \varrho)$ and $|D \theta|_{\infty ; a, \varrho} \leq 1$

$$
\begin{gathered}
\operatorname{spt}\left(\Phi_{\varepsilon} * \theta\right) \subset \mathbf{U}(a, \varepsilon+\varrho), \quad\left|T_{\varepsilon}(\theta)\right| \leq i(\varepsilon+\varrho)^{m+1} \leq i 2^{m+1} \varrho^{m+1} \quad \text { if } \varepsilon \leq \varrho, \\
\left(\operatorname{spt} R_{\varepsilon}\right) \cap\left\{x: \operatorname{dist}\left(x, A_{i}\right)<\varepsilon\right\}=\emptyset, \quad R_{\varepsilon}(\theta)=0 \quad \text { if } \varepsilon>\varrho, \\
\left|S_{\varepsilon}(\theta)\right| \leq\left|a_{\varepsilon} f_{\varepsilon}\right|_{\infty ; a, \varrho}|\theta|_{1 ; a, \varrho} \leq i 2^{m+1}|D \Phi|_{\infty ; 0,1} \boldsymbol{\alpha}(m) \varrho^{m+1} \\
\left|R_{\varepsilon}\right|_{-1,1 ; a, \varrho} \leq \gamma \varrho^{m+1} \quad \text { with } \gamma=2^{m+1} i\left(1+|D \Phi|_{\infty ; 0,1} \boldsymbol{\alpha}(m)\right),
\end{gathered}
$$

Now, A.1 may be applied with $A, R$ replaced by $A_{i}, R_{\varepsilon}$ to obtain

$$
\left|R_{\varepsilon}\right|_{-1,1 ; a, r} \leq \Gamma \gamma r \mathscr{L}^{m}\left(\mathbf{B}(a, 4 r) \sim A_{i}\right) \quad \text { for } 0<r<1 / i .
$$


Since $\mathbf{L}_{1}\left(\mathscr{L}^{m}, \mathbf{R}^{n-m}\right)$ is separable, one can use DS88, V.4.2, V.5.1, IV.8.3] to infer the existence of $S \in \mathscr{D}^{\prime}\left(\mathbf{R}^{m}, \mathbf{R}^{n-m}\right), f \in \mathbf{L}_{\infty}\left(\mathscr{L}^{m}, \mathbf{R}^{n-m}\right)$ and a sequence $\varepsilon_{j}$ with $\varepsilon_{j} \downarrow 0$ as $j \rightarrow \infty$ such that

$$
S(\theta)=\int f \bullet \theta \mathrm{d} \mathscr{L}^{m} \quad \text { for } \theta \in \mathscr{D}\left(\mathbf{R}^{m}, \mathbf{R}^{n-m}\right), \quad S_{\varepsilon_{j}} \rightarrow S \quad \text { as } j \rightarrow \infty .
$$

Defining $R=T-S$ and noting $R_{\varepsilon_{j}} \rightarrow R$ as $j \rightarrow \infty$,

$$
|R|_{-1,1 ; a, r} \leq \Gamma \gamma r \mathscr{L}^{m}\left(\mathbf{B}(a, 4 r) \sim A_{i}\right) \quad \text { for } 0<r<1 / i
$$

and [Fed69, 2.9.11] implies

$$
\lim _{r \rightarrow 0+} r^{-1-m}|R|_{-1,1 ; a, r}=0 \quad \text { for } \mathscr{L}^{m} \text { almost all } a \in A_{i}
$$

Moreover,

$$
\left|\int(f(x)-f(a)) \bullet \theta(x) \mathrm{d} \mathscr{L}^{m} x\right| \leq\left(\int_{\mathbf{U}(a, r)}|f(x)-f(a)| \mathrm{d} \mathscr{L}^{m} x\right) r|D \theta|_{\infty ; a, r}
$$

whenever $a \in A, 0<r<\infty, \theta \in \mathscr{D}\left(\mathbf{R}^{m}, \mathbf{R}^{n-m}\right)$ with $\operatorname{spt} \theta \subset \mathbf{U}(a, r)$ and [Fed69, 2.9.9] implies that one can take $T_{a}$ defined by $T_{a}(\theta)=\int \theta(x) \bullet$ $f(a) \mathrm{d} \mathscr{L}^{m} x$ for $\theta \in \mathscr{D}\left(\mathbf{R}^{m}, \mathbf{R}^{n-m}\right)$ for $\mathscr{L}^{m}$ almost all $a \in A_{i}$ in the existence part of the conclusion.

The uniqueness follows from 2.16 .

A.4 Remark. The splitting of $T$ into $S$ and $R$ was inspired by a similar procedure for functions used by Calderón and Zygmund in [CZ61, Theorem 7].

Max-Planck-Institute for Gravitational Physics (Albert-Einstein-Institute),

OT Golm, Am Mühlenberg 1, DE-14476 Potsdam, Germany

Ulrich.Menne@aei.mpg.de

\section{References}

[ADN59] S. Agmon, A. Douglis, and L. Nirenberg. Estimates near the boundary for solutions of elliptic partial differential equations satisfying general boundary conditions. I. Comm. Pure Appl. Math., 12:623727,1959 .

[ADN64] S. Agmon, A. Douglis, and L. Nirenberg. Estimates near the boundary for solutions of elliptic partial differential equations satisfying general boundary conditions. II. Comm. Pure Appl. Math., 17:35-92, 1964.

[All72] William K. Allard. On the first variation of a varifold. Ann. of Math. (2), 95:417-491, 1972.

[Alm68] F. J. Almgren, Jr. Existence and regularity almost everywhere of solutions to elliptic variational problems among surfaces of varying topological type and singularity structure. Ann. of Math. (2), 87:321$391,1968$. 
[Alm00] Frederick J. Almgren, Jr. Almgren's big regularity paper, volume 1 of World Scientific Monograph Series in Mathematics. World Scientific Publishing Co. Inc., River Edge, NJ, 2000. $Q$-valued functions minimizing Dirichlet's integral and the regularity of area-minimizing rectifiable currents up to codimension 2, With a preface by Jean E. Taylor and Vladimir Scheffer.

[AM03] Luigi Ambrosio and Simon Masnou. A direct variational approach to a problem arising in image reconstruction. Interfaces Free Bound., $5(1): 63-81,2003$.

[AS94] Gabriele Anzellotti and Raul P. Serapioni. $\mathcal{C}^{k}$-rectifiable sets. $J$. Reine Angew. Math., 453:1-20, 1994.

[Bra78] Kenneth A. Brakke. The motion of a surface by its mean curvature, volume 20 of Mathematical Notes. Princeton University Press, Princeton, N.J., 1978.

[Caf89] Luis A. Caffarelli. Interior a priori estimates for solutions of fully nonlinear equations. Ann. of Math. (2), 130(1):189-213, 1989.

[CZ61] Alberto P. Calderón and Antoli S. Zygmund. Local properties of solutions of elliptic partial differential equations. Studia Math., 20:171$225,1961$.

[DG61] Ennio De Giorgi. Frontiere orientate di misura minima. Seminario di Matematica della Scuola Normale Superiore di Pisa, 1960-61. Editrice Tecnico Scientifica, Pisa, 1961.

[DS88] Nelson Dunford and Jacob T. Schwartz. Linear operators. Part I. Wiley Classics Library. John Wiley \& Sons Inc., New York, 1988. General theory, With the assistance of William G. Bade and Robert G. Bartle, Reprint of the 1958 original, A Wiley-Interscience Publication.

[EG92] Lawrence C. Evans and Ronald F. Gariepy. Measure theory and fine properties of functions. Studies in Advanced Mathematics. CRC Press, Boca Raton, FL, 1992.

[Fed69] Herbert Federer. Geometric measure theory. Die Grundlehren der mathematischen Wissenschaften, Band 153. Springer-Verlag New York Inc., New York, 1969.

[Giu03] Enrico Giusti. Direct methods in the calculus of variations. World Scientific Publishing Co. Inc., River Edge, NJ, 2003.

[GT01] David Gilbarg and Neil S. Trudinger. Elliptic partial differential equations of second order. Classics in Mathematics. Springer-Verlag, Berlin, 2001. Reprint of the 1998 edition.

[Hut86] John E. Hutchinson. Second fundamental form for varifolds and the existence of surfaces minimising curvature. Indiana Univ. Math. J., $35(1): 45-71,1986$. 
[LO77] H. B. Lawson, Jr. and R. Osserman. Non-existence, non-uniqueness and irregularity of solutions to the minimal surface system. Acta Math., 139(1-2):1-17, 1977.

[Man96] Carlo Mantegazza. Curvature varifolds with boundary. J. Differential Geom., 43(4):807-843, 1996.

[Men09a] Ulrich Menne. Some applications of the isoperimetric inequality for integral varifolds. Adv. Calc. Var., 2:247-269, 2009.

[Men09b] Ulrich Menne. Decay estimates for the quadratic tilt-excess of integral varifolds, 2009. arXiv:0909.3253v2 [math.DG], submitted.

[Men10] Ulrich Menne. A Sobolev Poincaré type inequality for integral varifolds. Calc. Var. Partial Differential Equations, 38:369-408, 2010.

[Mor66] Charles B. Morrey, Jr. Multiple integrals in the calculus of variations. Die Grundlehren der mathematischen Wissenschaften, Band 130. Springer-Verlag New York, Inc., New York, 1966.

[MR09] Luca Mugnai and Matthias Röger. Convergence of perturbed Allen Cahn equations to forced mean curvature flow, 2009. arXiv: 0902.1816v1 [math. AP].

[Reš68] Yurii G. Rešetnyak. Generalized derivatives and differentiability almost everywhere. Math. USSR, Sb., 4:293-302, 1968.

[Rög04] Matthias Röger. Solutions for the Stefan problem with GibbsThomson law by a local minimisation. Interfaces Free Bound., 6(1):105-133, 2004.

[RS06] Matthias Röger and Reiner Schätzle. On a modified conjecture of De Giorgi. Math. Z., 254(4):675-714, 2006.

[Sch01] Reiner Schätzle. Hypersurfaces with mean curvature given by an ambient Sobolev function. J. Differential Geom., 58(3):371-420, 2001.

[Sch04] Reiner Schätzle. Quadratic tilt-excess decay and strong maximum principle for varifolds. Ann. Sc. Norm. Super. Pisa Cl. Sci. (5), 3(1):171-231, 2004.

[Sch09] Reiner Schätzle. Lower semicontinuity of the Willmore functional for currents. J. Differential Geom., 81(2):437-456, 2009.

[Sim83] Leon M. Simon. Lectures on geometric measure theory, volume 3 of Proceedings of the Centre for Mathematical Analysis, Australian National University. Australian National University Centre for Mathematical Analysis, Canberra, 1983.

[Sim97] Leon Simon. Schauder estimates by scaling. Calc. Var. Partial Differential Equations, 5(5):391-407, 1997.

[Tru89] Neil S. Trudinger. On the twice differentiability of viscosity solutions of nonlinear elliptic equations. Bull. Austral. Math. Soc., 39(3):443447, 1989. 
[Wlo87] J. Wloka. Partial differential equations. Cambridge University Press, Cambridge, 1987. Translated from the German by C. B. Thomas and M. J. Thomas.

[Zie89] William P. Ziemer. Weakly differentiable functions, volume 120 of Graduate Texts in Mathematics. Springer-Verlag, New York, 1989. Sobolev spaces and functions of bounded variation. 